\title{
Philosophiques
}

\section{Au-delà et en deça de l'homme social : les pôles de la pensée de Rousseau}

\section{Gérard Allard}

Volume 14, numéro 2, automne 1987

URI : https://id.erudit.org/iderudit/027014ar

DOI : https://doi.org/10.7202/027014ar

Aller au sommaire du numéro

Éditeur(s)

Société de philosophie du Québec

ISSN

0316-2923 (imprimé)

1492-1391 (numérique)

Découvrir la revue

Citer cet article

Allard, G. (1987). Au-delà et en deça de l'homme social : les pôles de la pensée de Rousseau. Philosophiques, 14(2), 264-298. https://doi.org/10.7202/027014ar
Résumé de l'article

La pensée de Jean-Jacques Rousseau est essentiellement une pensée politique, qui oscille entre un pôle transpolitique, que le citoyen de Genève incarne, par exemple, dans les personnages du législateur, du pédagogue et du sage, et un pôle prépolitique, où figurent l'homme naturel, le marginal et l'homme sensible. C'est en examinant quelques pages de trois grandes oeuvres de Rousseau: l'« Emile », « Du contrat social » et " Julie ou la Nouvelle Héloïse », que je tenterai d'éclaircir brièvement ces points. Ainsi sera manifestée l'actualité des paradoxes rousseauistes. 


\title{
AU-DELÀ ET EN DEÇA DE L'HOMME SOCIAL : LES PÔLES DE LA PENSÉE DE ROUSSEAU
}

\author{
par Gérald Allard
}

\begin{abstract}
RÉSUMÉ. La pensée de Jean-Jacques Rousseau est essentiellement une pensée politique, qui oscille entre un pôle transpolitique, que le citoyen de Genève incarne, par exemple, dans les personnages du législateur, du pédagogue et du sage, et un pôle prépolitique, où figurent l'homme naturel, le marginal et l'homme sensible. C'est en examinant quelques pages de trois grandes œuvres de Rousseau: l'«Émile », « Du contrat social » et «Julie ou la Nouvelle Héloïse», que je tenterai d'éclaircir brièvement ces points. Ainsi sera manifestée l'actualité des paradoxes rousseauistes.
\end{abstract}

ABSTRACT. Rousseau's thought is essentially a political thought that swings from a transpolitical pole, presented through the figures of the legislator, the pedagogue and the wise man, and a prepolitical pole, where we meet natural man, the outsider and the man of exquisite sensibility. This theme is brought to light through a partial analysis of three of Rousseau's major works : «Emile», «Du contrat social » and the «Nouvelle Héloïse ». In this way, it is hoped that Rousseau's paradoxes will be shown to have meaning for our contemporaries.

«...Épicure et Montaigne...Platon et Rousseau. C'est avec eux qu'il faut que je m'explique, lorsque j'ai longtemps cheminé solitaire, c'est par eux que je veux me faire donner tort et raison, et je les écouterai lorsque, devant moi, ils se donnent tort et raison les uns aux autres."

(Friedrich NieTzSCHE) 


\section{Introduction}

Jean-Jacques Rousseau fut d'abord et avant tout un philosophe politique, ou, selon certains, un penseur politique. C'est ce qu'exprime l'opinion commune, laquelle ramène sa pensée aux deux thèses que «l'homme est naturellement bon» et surtout que «c'est la société qui le rend méchant». D'ailleurs, Rousseau lui-même cautionne cette simplification, par exemple, dans la Lettre $\dot{a}$ Christophe de Beaumont : cherchant à faire l'histoire de sa pensée, il fixe son étonnement originel et son scandale constant dans la thèse centrale de l'opposition chez l'homme entre l'être, c'est-àdire le bien, et le paraître, c'est-à-dire le mal ; il aurait découvert que la racine de cette opposition est le système social et donc que le remède humain par excellence est politique ${ }^{1}$. De même, dans Les Confessions il déclare que le Contrat social, ou plutôt l'œuvre dont il est tiré et qu'il ne termina jamais, les Institutions politiques, sont son magnum opus ${ }^{2}$. C'est pourquoi le titre de gloire de Rousseau, à savoir d'être citoyen de Genève plutôt que pur et simple philosophe, prend son sens plein du fait que sa réflexion philosophique est justement politique plutôt que métaphysique, logique ou autre ${ }^{3}$.

1. Sitôt que je fus en état d'observer les hommes, je les regardois faire, et je les écoutois parler; puis, voyant que leurs actions ne ressembloient point à leurs discours, je cherchai la raison de cette dissemblance, et je trouvai qu'être et paroître étant pour eux deux choses aussi différentes qu'agir et parler, cette deuxième différence étoit la cause de l'autre, et avoit elle-même une cause qui me restoit à chercher. Je la trouvai dans notre ordre social, qui, de tout point contraire à la nature que rien ne détruit, la tirannise sans cesse, et lui fait sans cesse réclamer ses droits. Je suivis cette contradiction dans ses conséquences, et je vis qu'elle expliquoit seule tous les vices des hommes et tous les maux de la société. » Lettre à Christophe de Beaumont, Pléiade IV, pages 966 967. Voir aussi Émile, Pléiade IV, page 524. - Toutes les citations de l'œuvre de Rousseau sont tirées de l'édition de la Bibliothèque de la Pléiade. L'indication « Pléiade IV », par exemple, indique que le texte se trouve dans le quatrième volume de ladite édition.

2. "Des divers ouvrages que j'avois sur le chantier, celui que je méditois depuis plus longtemps, dont je m'occupois avec le plus de gout, auquel je voulois travailler toute ma vie, et qui devoit selon moi mettre le sceau à ma réputation étoit mes Institutions politiques... J'avois vu que tout tenoit radicalement à la politique, et que, de quelque façon qu'on s'y prit, aucun peuple ne seroit jamais que ce que la nature de son Gouvernement le feroit être ; ainsi cette grande question du meilleur Gouvernement possible me paraissoit se réduire à celle-ci. Quelle est la nature de Gouvernement propre à former un Peuple le plus vertueux, le plus éclairé, le plus sage, le meilleur enfin à prendre ce mot dans son plus grand sens. "Les Confessions, Pléiade I, pages 404-405

3. "Voici une des grandes et des plus belles questions qui ayent jamais été agitées. Il ne s'agit point dans ce Discours de ces subtilités métaphysiques qui ont gagné toutes les 
On trouvera sans difficulté chez Rousseau une anthropologie, les principes d'une épistémologie, des réflexions sur la divinité et bien d'autres considérations appartenant aux domaines mieux connus de la philosophie ; n'empêche que toute son œuvre gravite autour d'un centre qui est politique ${ }^{4}$. Il faut ajouter cependant que cette pensée, tout en étant politique de part en part, est pour l'essentiel une remise en question des plus radicales du politique.

Car si Rousseau est un penseur politique, il est aussi un auteur à paradoxes, c'est-à-dire un penseur qui, par une nécessité tout autant que par certains éléments de son système, pense à côté (ce qui se dit "para » en grec) des opinions reçues (soit «doxai» en grec). Rousseau prend un malin plaisir à contredire les opinions courantes, fût-ce les opinions qu'il a lui-même exprimées ou soutenues. C'est du moins l'accusation qu'on porta contre lui dès le début de sa carrière et qu'on reprit constamment jusqu'à la fin de sa vie. Mais il ne saurait suffire de dire qu'un penseur a proféré un paradoxe pour prouver qu'il a tort : c'est une méthode philosophiquement bien inadéquate, quoique dangereusement efficace, que de souligner les présuppositions ou conséquences étonnantes d'une thèse dans le but de la déprécier aux yeux d'un certain public. Rousseau se plaignait déjà d'être traité ainsi dans une réponse qu'il fit à une des nombreuses «réfutations » de son Premier Discours: "Il y a des vérités très-certaines qui au premier coup d'œil paroissent des absurdités, et qui passeront toujours pour telles auprès de la plûpart des gens... Il en est ainsi du sentiment que je soutiens. Les hommes les plus superficiels ont toujours été les plus

parties de la Littérature, et dont les Programmes d'Académie ne sont pas toujours exemps ; mais il s'agit d'une de ces vérités qui tiennent au bonheur du genre humain. » Premier Discours, Pléiade III, page 3. - «Né citoyen d'un État libre, et membre du souverain, quelque foible influence que puisse avoir ma voix dans les affaires publiques, le droit d'y voter suffit pour m'imposer le devoir de m'en instruire. Heureux, toutes les fois que je médite sur les Gouvernements, de trouver toujours dans mes recherches de nouvelles raisons d'aimer mon pays ! " Du contrat social, Pléiade III, page 351 .

4. C'est par exemple l'opinion qu'exprime Victor GoLDSCHMIDT à la fin de son admirable livre: Anthropologie et politique : les principes du système de Rousseau, Librairie philosophique J. Vrin, Paris, 1974, pages 779-781. - $\grave{A}$ la suite de Goldschmidt, il faut sans doute «corriger» cette première affirmation en signalant que la pensée politique de Rousseau est fondée elle-même dans une anthropologie. Mais il n'en demeure pas moins que le point de vue de l'auteur est avant tout, et après cout, politique. 
prompts à prendre parti contre moi; les vrais Philosophes se hâtent moins ; et si j'ai la gloire d'avoir fait quelques prosélytes ; ce n'est que parmi ces derniers 5 . " Au fond, l'insatisfaction de Rousseau vient de ce qu'il espère s'adresser à des penseurs, à des hommes qui ne sont pas repoussés comme mécaniquement par une opinion qui est à côté de l'opinion reçue : par ces hommes d'exception, Rousseau s'attend à être entendu avant d'être jugé, quelque étrange que puisse paraître sa position au premier abord ${ }^{6}$. Espoir et attente souvent déçus.

Par ailleurs - conséquence inversée de ses déceptions -, Rousseau se targue justement d'être paradoxal : c'est que la vérité, telle qu'il la perçoit, ne peut être autrement que hors des bornes du «bon sens" et donc par-delà les avis de tout un chacun ${ }^{7}$. Cette nécessité tient à au moins deux données qu'il faut rapprocher : les limites du langage et les limites des hommes. Les aspects du réel et, conséquemment, les idées nécessaires pour les exprimer dépassent largement la maigre mesure pratique de la capacité de nommer de

5. Dernière réponse de J.-J. Rousseau de Genève, Pléiade III, page 71. Comparer la citation au passage suivant de la Lettre à Christophe de Beaumont, Pléiade IV, page 928 : «Après mon premier Discours, j'étois un homme à paradoxes, qui se faisoit un jeu de prouver ce qu'il ne pensoit pas... ” - Par ailleurs, Rousseau traita souvent les autres penseurs tout aussi injusternent qu'il se plaignait d'être traité : tout en étant un lecteur fort perspicace et judicieux des grands philosophes avec lesquels il entretenait un vigoureux dialogue : les Locke, les Hobbes et autres, il ne se gênait pas pour embrigader le «bon sens» et l'indignation pour mieux assurer la victoire des thèses qu'il croyait les plus vraies et ce au mépris d'une certaine vérité. Voir à ce sujet la quatrième promenade dans Les Rêveries du promeneur solitaire (Pléiade I, pages 1024-1039) où Rousseau expose une théorie passablement «sophistiquée » de la véracité.

6. Il est probable que la célèbre cassure qui se fit entre Rousseau et ses amis encyclopédistes et savants est due en partie au fait que ces derniers n'avaient pas bien perçu la sincérité de leur confrère : sans doute, s'imaginaient-ils que sa prise de position, qui, en dernière analyse, remettait en cause tout le mouvement des «Lumières» dont ils étaient les partisans, n'était justement qu'un paradoxe, $c$ 'est-à-dire une espèce de jeu intellectuel visant à se faire connaître et reconnaître dans la jungle littéraire française : il devait leur paraitre inconcevable qu'un des leurs puisse remettre en doute le dogme fondamental de leur coterie. Avec le temps, voyant Rousseau persister dans son opinion, ils ont dû peu à peu déchanter et reconnaître qu'il croyait tout de bon ce qu'il écrivait : c'est alors que la lutte avec ce «barbare» - c'est ainsi que Rousseau se présente à son lecteur dans l'épigraphe du Premier Discours - prit pour eux tout son sérieux, d'autant plus qu'enfin détrompés ils devaient se sentir dupés.

7. «Lecteurs vulgaires, pardonnez-moi mes paradoxes. Il en faut faire quand on réfléchit, et quoi que vous puissiez dire, j'aime mieux être homme à paradoxes qu'homme à préjugés. » Émile. Pléiade IV, page 323. 
l'homme. Cela est manifeste par exemple dans l'analogie : par une raisonnable économie instrumentale, un seul mot est utilisé pour signifier des réalités distinctes mais apparentées. L'analogie, stratégie langagière inévitable et même, à plusieurs titres, éminemment souhaitable, conduira un esprit non averti ou peu délié à voir des identités là où il n'y a que des ressemblances; aussi, elle le conduira à s'imaginer découvrir une contradiction dans les idées d'un penseur qui, lui, perçoit les différences derrière l'unité nominale et en tient compte ${ }^{8}$. Cette supposée contradiction, une fois « débusquée », est dénoncée sous l'appellation de paradoxe. Ensuite, pour Rousseau, la vérité n'est pas facilement accessible: les hommes ont suppléé à la carence du vrai par des approximations plus ou moins heureuses, pour ne pas dire des erreurs grossières. Ce qui revient à supposer que les hommes vivent comme plus naturellement dans l'opinion vraisemblable mais fausse que dans la vérité. À quoi s'ajoute que dans l'état de société le mensonge pieux, ou politique, est un expédient efficace et nécessaire. Dans un tel contexte, les affirmations de celui qui voit clair et qui tente de rendre les autres clairvoyants paraissent, du moins dans un premier temps, comme autant de folies, d'insanités, de rêveries impraticables, c'est-à-dire, encore une fois, comme des paradoxes ${ }^{9}$.

8. “J'ai fait cent fois réflexion en écrivant qu'il est impossible dans un long ouvrage de donner toujours les mêmes sens aux mêmes mots. Il n'y a point de langue assés riche pour fournir autant de termes, de tours et de phrases que nos idées peuvent avoir de modificarions... Malgré cela, je suis persuadé qu'on peut être clair, même dans la pauvreté de norre langue; non pas en donnant toujours les mêmes acceptions aux mêmes mots, mais en faisant en sorte, autant de fois qu'on employe chaque mot, que l'acception qu'on lui donne soit suffisament déterminée par les idées qui s'y rapportent, et que chaque période où ce mot se trouve lui serve, pour ainsi dire, de définition... je ne crois pas en cela me contredire dans mes idées, mais je ne puis disconvenir que je ne me contredise souvent dans mes expressions. » Émile, Pléiade IV, page 345. - Comme en fait foi son utilisation de mots comme « liberté", " nature » et " vertu», Rousseau est un maître de l'analogie.

9. À cet effet, on consultera la Fiction ou morceau allégorique sur la révélation (Pléiade IV, pages 1044-1054). Ce texte peu connu montre au moyen des figures d'un Socrate et d'un Christ, d'abord, le problème et les dangers qu'implique la communication de la vérité à des gens prévenus contre elle et, ensuite, une partie de la solution qu'imagina Rousseau : la rhétorique. Pour éviter les nombreux barrages sociaux et psychologiques dressés contre la vérité, pour exprimer sur un plan tout émotif et donc efficace parce que immédiatement perceptible l'essentiel d'une pensée, Rousseau croit que le ton de voix et l'émotion qu'elle porte sont les meilleurs moyens : les seuls mots, la seule raison ne suffisent plus, n'ont jamais suffi quand il s'agit de lutter contre les prestiges de l'erreur et de la socialisation, contre les moyens pratiques des hommes au pouvoir, et reconduire les hommes auprès d'eux-mêmes. Voilà pourquoi il insiste souvent sur le 
C'est à la lumière de ces remarques qu'on peut comprendre les nombreuses fois où Rousseau se fait provocateur et affiche ses paradoxes comme autant de preuves de l'exactitude de sa pensée : "À l'égard de ce qu'on appellera la partie sistématique, qui n'est autre chose ici que la marche de la nature, c'est-là ce qui déroutera le plus le Lecteur ; c'est aussi par-là qu'on m'attaquera sans doute ; et peut-être n'aura-t-on pas tort. On croira moins lire un Traité d'éducation que les rêveries d'un visionnaire sur l'éducation. Qu'y faire? Ce n'est pas sur les idées d'autrui que j'écris ; c'est sur les miennes. Je ne vois point comme les autres hommes; il y a longtemps qu'on me l'a reproché ${ }^{10}$. » Tout le drame de la vie du citoyen de Genève, et jusqu’à sa paranoïa, peut s'entrevoir dans ces remarques «épistémologiques ». Car si dire la vérité implique la production quasi systématique de paradoxes, la vie consacrée à la vérité ${ }^{11}$ ne peut être que paradoxale : le vrai philosophe est, par définition, une pierre d'achoppement.

Les œuvres de Jean-Jacques Rousseau abondent donc en paradoxes. Pourtant, en un sens, au-delà de la diversité manifeste, c'est toujours le même problème contre lequel achoppe le lecteur. Il comprend mal comment l'auteur peut se permettre une espèce de «bi-localisation » intellectuelle. Il se demande comment Rousseau peut prétendre se situer à la fois ici et là, c'est-à-dire comment il peut simultanément prendre une position et en soutenir une autre qui est aux antipodes. Donnons quelques exemples de cette «bilocalisation » problématique, exemples tirés des œuvres les mieux connues. Comment le Premier Discours, qui remet en question la validité morale et l'utilité politique des sciences et des arts, peut-il sortir de la plume d'un homme qui, selon l'évidence du texte luimême, est un familier des belles-lettres ? Peut-on simultanément

ton de ses propres œuvres. Voir, par exemple, Les Confessions, Pléiade I, pages 502 er 623. - On peut dire que la froideur ou la dureté des écrits modernes est pour Rousseau à la fois une preuve de leur inexactitude "théorique» de base et la cause de leur inefficacité finale. C'est le sens de son analyse de la langue naturelle de l'homme et le fondement de son jugement sur la langue moderne. Voir l'Essai sur l'origine des langues, Cabiers pour l'Analyse, Paris, Le Graphe, 1970.

10. Émile, Pléiade IV, page 242. - On examinera aussi, entre autres, les préfaces au Premier Discours (Pléiade III, page 3) et au Second Discours (Pléiade III, page 125).

11. Voir encore une fois la quatrième promenade des Rêveries (Pléiade I, page 1024), où Rousseau fait son examen de conscience à la lumière de sa devise : «Consacrer sa vie à la vérité ». - $A$ noter comment Rousseau a complètement renversé le sens original de cette citation de Juvénal (Satires, IV. 91). 
prétendre être un adepte des belles-lettres et se vouloir leur condamnateur ${ }^{12}$ ? Ou encore : comment l'auteur du Second Discours peut-il faire l'apologie d'un hypothétique état de nature et puis d'un état de sauvagerie, où la rationalité est une impossibilité ou peu s'en faut, alors que ce même auteur, pour développer et appuyer sa thèse, utilise toutes les ressources de l'argumentation rationnelle, de la documentation scientifique et des artifices de l'art d'écrire ${ }^{13}$ ? Ensuite : comment dans la Nouvelle Héloïse, le romancier peut-il brosser le tableau de l'amour humain le plus tendre et de la fidélité maritale la plus déterminée, alors que JeanJacques Rousseau fit tout le contraire durant une vie assez mouvementée où, de son propre aveu, il connut par faiblesse quelques aventures sexuelles avant et après avoir conçu cinq enfants avec une femme qu'il n'aimait probablement pas vraiment? Et comment l'Émile peut-il offrir les réflexions pédagogiques de ce même homme qui fut chassé de l'emploi de précepteur, qui reconnaît ne pas posséder les talents du rôle et qui abandonna ses propres enfants aux Enfants-Trouvés? Dernier exemple: les Dialogues, comme le signale Jean Starobinski, sont des chefs-d'cuvre de raisonnement mis au service du rejet de la réflexion, des sommets de littérature créés pour nier la littérature. «L'ouvrage tout entier est une réflexion malheureuse et honteuse, fascinée par la nostalgie de l'irréfléchi: elle se condamne et se renie elle-même en se développant, et du même coup elle aggrave et prolonge la faute d'écrire et de réfléchir, dont Rousseau se dit innocent ${ }^{14}$. » Ne voiton pas, demanderont certains, apparaître et réapparaître, dans chacun de ces exemples, la même contradiction qui mine l'œuvre philosophique depuis ses débuts et jusqu'en ses derniers avatars? On dirait que Rousseau se plaît à produire des œuvres qui offrent

12. Préface au Narcisse, Pléiade II, page 961.

13. Comparer, par exemple, l'auteur qui affirme: «Mon sujet intéressant l'homme en général, je tâcherai de prendre un langage qui convienne à toutes les Nations, ou plutôt, oubliant les tems et les Lieux, pour ne songer qu'aux Hommes à qui je parle, je me supposerai dans le Licée d'Athenes, repetant les Leçons de mes Maitres, ayant les Platons et les Xenocrates pour Juges, et le Genre-humain pour Auditeur (Second Discours, Pléiade III, page 133).»; et l'homme décrit deux pages plus loin : «... Je le (c'est-à-dire l'homme dans l'état de nature) vois se rassasiant sous un chesne, se désalterant au premier Ruisseau, trouvant son lit au pied du même arbre qui lui a fourni son repas, et voilà ses besoins satisfaits (page 135). »

14. Jean-Jacques Rousseau : la transparence et l'obstacle, NRF, Éditions Gallimard, 1971, p. 252. 
le contraste le plus parfait mais le plus déconcertant avec la vie et le statut social et moral de l'ouvrier. Tous connaissent la trop fameuse paranoïa de Rousseau. Mais n'y aurait-il pas lieu d'ajouter à son dossier médical et de parler de sa schizophrénie?

\section{Le citoyen, le législateur et l'autre}

Jusqu'ici nous avons souligné un paradoxe constant de l'œuvre de Rousseau surgissant d'une «contradiction» entre le lieu de l'auteur et la position intellectuelle qu'il défend ou propose. Mais à l'intérieur même des thèses du philosophe de Genève, on découvre une seconde "bi-localisation» ou même "tri-localisation», plus importante peut-être parce qu'elle tient justement à la doctrine rousseauiste en elle-même : découverte qui permet de "reprocher » à Rousseau de donner deux, ou même trois, solutions séparées et opposées aux problèmes politiques, pédagogiques et moraux qu'il soulève. En règle générale, peut-on dire, tout en faisant l'apologie de la vertu civile et sociale la plus traditionnelle, il suggère à ses lecteurs que le bonheur et l'excellence se situent dans une vie qui dépasse la condition humaine ordinaire ou, tout au contraire, dans une existence qui ne réussit pas à atteindre le minimum qu'on attribue en propre à l'humanité : les pôles de la pensée politique de Rousseau sont fixés au-delà et en deçà de la vie du citoyen. On comprendra sans doute mieux ce qui est proposé ici lorsqu'on aura examiné quelques exemples. Du même coup, ce début d'induction permettra de deviner qu'on atteint ici une structure de base de l'œuvre et de la pensée de Rousseau.

Le Contrat social est sans doute le livre de Rousseau qui est aujourd'hui le mieux connu et le plus commenté : contrairement à la Nouvelle Héloïse ou à l'Émile, par exemple, il n'a pas souffert du changement des goûts qui traite de romantisme larmoyant le style de Rousseau; il se trouve par ailleurs être à l'origine de bon nombre de thèses politiques placées aujourd'hui parmi les évidences aveuglantes ou les progrès les plus importants de la modernité. L'objet de ce petit traité est de découvrir le fondement institutionnel de la vie sociale et politique et d'esquisser le plan de ce qu'on peut y construire conformément aux règles du droit naturel. "Je veux chercher si dans l'ordre civil il peut y avoir quelque règle d'administration légitime et sûre, en prenant les hommes tels qu'ils sont, 
et les loix telles qu'elles peuvent être : Je tâcherai d'allier toujours dans cette recherche ce que le droit permet avec ce que l'intérêt prescrit, afin que la justice et l'utilité ne se trouvent point divisées ${ }^{15}$. " Ici il n'est pas d'abord question de dénoncer un abus, comme dans le Premier Discours, ou de découvrir les racines anthropologiques de la morale et de la politique, comme dans le Second Discours : le sujet du livre est campé solidement et, croirait-on, exclusivement dans le champ du politique.

On résumera, sans trop craindre de se tromper, la thèse de Rousseau en parlant du pacte social et de la volonté générale: l'origine de la société, et le constant point de référence de la vie en société, est la volonté conjuguée de tous les citoyens. Cette thèse suppose que les hommes sont naturellement égaux et libres et que cette égalité et cette liberté trouvent leurs source et fondement dans leur nature, pour ne pas dire dans l'état de nature. Or à un moment donné de l'histoire d'un groupe, ces propriétés primordiales de l'homme sont menacées par les circonstances ; elles deviennent physiquement, ou plus exactement biologiquement, impossibles. C'est pourquoi il faut trouver un nouveau mode de vie qui sache assurer autant que possible ces biens naturels de base. Par un paradoxe tout rousseauiste, la solution politique de cette impasse est l'aliénation totale de la liberté individuelle au groupe qui se constitue du fait même de cette aliénation : c'est cet artifice qui porte le nom de contrat social. "Ces clauses bien entendues se réduisent toutes à une seule, savoir l'aliénation totale de chaque associé avec tous ses droits à toute la communauté : Car premièrement, chacun se donnant tout entier, la condition est égale pour tous, et la condition étant égale pour tous, nul n'a intérêt de la rendre onéreuse aux autres ${ }^{16}$. » Semblablement, par un acte de la volonté générale qui crée une loi, ou plus exactement qui est une loi, l'être humain transformé en citoyen n'agit plus comme individu, ni ne se préoccupe de lui-même en tant qu'individu distinct et opposé à tous les autres. En d'autres termes, à l'instar du contrat social, une loi véritable est l'expression de volontés unanimes, où chaque être humain commande à tous les membres de son groupe sans exception et, du même coup, à soi-même à partir de sa perception de ce qu'il lui serait nécessaire pour survivre et bien

15. Du contrat social, Pléiade III, page 351.

16. Ibid., pages 360-361. 
vivre; inversement, toute décision pratique universelle dans son origine et dans sa portée est une bonne loi ${ }^{17}$. Dans la cité fondée sur le contrat social - et d'une façon ou de l'autre, toute société repose ultimement sur cette base - , la liberté individuelle naturelle n'existe plus ; c'est là, dirait Rousseau, le prix de l'égalité et de la liberté civiles et de la justice, voire de la liberté morale et du progrès des lumières ${ }^{18}$. L'individu appellé citoyen se définit par rapport au tout dont il fait partie : qui dit citoyen dit cité, et qui dit cité dit tout institutionnel qui annihile le tout naturel que constituait l'individu originel.

Tout comme l'indépendance naturelle qui disparaît par définition dans la cité pour renaître sous la forme de la participation à la souveraineté et aussi de l'autarcie du groupe, l'égalité naturelle est supposée demeurer en se transformant en égalité entre les citoyens. Aussi le lecteur du Contrat social ne peut manquer d'être surpris par deux exceptions à l'exigeante règle d'unanimité qu'imposent le pacte social et la volonté générale, et du même coup, fautil croire, à la règle de l'égalité. La première, la plus frappante, la plus souvent commentée de ces exceptions est évidemment le législateur qui fait une apparition inopinée dans le deuxième livre du traité. Reconnaissant que les petites gens qui forment la très grande majorité des membres du souverain politique n'ont pas l'intelligence requise pour imaginer et formuler les lois les plus prudentes, c'est-à-dire celles qui assureraient le mieux leur sécurité et leur bonheur politique, l'auteur est conduit, par les conditions de possibilité d'un régime légitime, à consacrer un chapitre entier à un être humain on ne peut plus spécial qui fait, pour ainsi dire, le pont entre le bon peuple et la bonne législation ${ }^{19}$.

17. «Ainsi par la nature du pacte, tout acte de souveraineté, c'est-à-dire tout acte authentique de la volonté générale, oblige ou favorise également tous les Citoyens, ensorte que le Souverain connoit seulement le corps de la nation et ne distingue aucun de ceux qui la composent. "Ibid., page 374. - Pour ce qui est de la thèse que la volonté générale est nécessairement bonne, voir le troisième chapitre du deuxième livre.

18. Ibid., pages 364-365, 391 .

19. Voir le septième chapitre du deuxième livre du Contrat social. Noter que ce chapitre intitulé «Du législateur» se situe entre les chapitres intitulés «De la loi.»et «Du peuple. ». - "De lui-même le peuple veut toujours le bien, mais de lui-même il ne le voit pas toujours. La volonté générale est toujours droite, mais le jugement qui la guide n'est pas toujours éclairé. Il faut lui faire voir les objets tels qu'ils sont, quelquefois tels qu'ils doivent lui paroitre, lui montrer le bon chemin qu'elle cherche, la garantir de la séduction des volontés particulières, rapprocher à ses yeux les lieux et les tems, 
Le législateur est un être spécial à plus d'un titre. D'abord, son œuvre essentielle est, aux yeux de Rousseau, à peine imaginable en raison de la nature même du pacte social: le législateur doit neutraliser la nature humaine dans ce qu'elle a de plus radical pour lui substituer une nouvelle constitution artificielle, sans pourtant anéantir le droit naturel ni même la possibilité de retrouver un état pré-social. «Celui qui ose entreprendre d'instituer un peuple doit se sentir en état de changer, pour ainsi dire, la nature humaine; de transformer chaque individu, qui par lui-même est un tout parfait et solitaire, en partie d'un plus grand tout dont cet individu reçoive en quelque sorte sa vie et son être; d'altérer la constitution de l'homme pour la renforcer; de substituer une existence partielle et morale à l'existence physique et indépendante que nous avons tous reçue de la nature ${ }^{20}$. $)$ Or, pour réaliser ce miracle de fondation politique et de transformation anthropologique, le législateur se trouve tout à fait à l'extérieur du circuit politique qui aurait pu accroître ses forces et rendre son « miracle » faisable. Il lui faudrait être effectivement et visiblement plus puissant que la nature, mais il ne peut être qu'un homme placé devant une foule d'autres. Car il n'est pas membre du souverain et, en conséquence, ne peut même pas voter pour établir la constitution qu'il a imaginée : «Celui qui rédige les loix n'a donc ou ne doit avoir aucun droit législatif... ${ }^{21}$. Mais il n'est pas non plus un prince qui institue un régime : il est le précepteur du prince; d'ailleurs, instrument plutôt que source du pouvoir, le prince, dans la pensée de Rousseau, est engendré par le peuple, une fois que ce dernier s'est institué souverain et a choisi son régime ${ }^{22}$. Ni membre du souverain, ni membre du gouvernement, mais rouage indispensable à l'existence légitime des deux seules parties du corps politique, le législateur est et n'est pas partie intégrante de la théorie politique de Rousseau.

balancer l'attrait des avantages présens et sensibles, par le danger des maux éloignés et cachés... Voilà d'où naît la nécessité d'un Législateur.»Ibid., page 380. - L'italicisation n'est pas dans l'original.

20. Ibid., page 381 - Voir aussi, au sujet de ce trait fondamental de la nature humaine et de sa déformation dans l'état social, Émile, Pléiade IV, page 249. - Pour ce qui est de la possibilité rémanente de se soustraire au contrat, voir plus bas.

21. Ibid., page 383 .

22. Voir Ibid., pages 381 et 399 . - Bien mieux: l'ouvre du législateur, se situant par définition avant le pacte social ou, du moins, avant la législation fondamentale, il n'y a à proprement parler aucun souverain vraiment légitime ni aucun gouvernement vraiment actif dont le législateur pourrait espérer puiser les énergies nécessaires à son aeuvre. 
Il y a de quoi se demander finalement si le législateur est vraiment un être humain. Serait-il un dieu alors? Peut-être ; c'est du moins ce que suggère l'auteur du Contrat social. Mais, pour coller aux idées ou phantasmes de Rousseau, on ferait mieux de parler d'un être à deux natures : une sorte d'hybride qui connaisse la nature humaine comme de l'intérieur, mais qui ne soit sujet à aucune de ses faiblesses, qui soit au-delà de son individualité propre, mais qui sonde très exactement tous les particuliers. «Pour découvrir les meilleures règles de société qui conviennent aux Nations, il faudroit une intelligence supérieure, qui vit toutes les passions des hommes et qui n'en éprouvât aucune, qui n'eut aucun rapport avec notre nature et qui la connaît à fond, dont le bonheur fût indépendant de nous et qui pourtant voulut bien s'occuper du notre; enfin qui, dans le progrès des tems se ménageant une gloire éloignée, put travailler dans un siècle et jouir dans un autre. Il faudrait des Dieux pour donner des loix aux hommes ${ }^{23}$. 》 La contradictoire dualité que détaille ce texte fait croire que Rousseau parle d'une législation, pour ainsi dire, révélée et d'un législateur humain qui participe d'une quelconque façon de la nature divine. La fin du même chapitre, qui reprend la thèse machiavélienne du mensonge fondateur, le législateur cautionnant son enseignement à coup d'autorité divine et de menaces de sanctions eschatologiques, permet de comprendre comment il faut entendre ce passage crucial, du moins quant à ses implications pratiques essentielles ${ }^{24}$. Il n'est pas difficile d'entrevoir quel contemporain Rousseau a en tête lorsqu'il parle de ces êtres qui transcendent l'opinion des citoyens et les limires des sociétés particulières : il faut se croire législateur en puissance pour légiférer aussi sûrement sur leur œuvre. Mais, capable de dire ce qu'il fallait faire, Rousseau savait mieux que personne qu'il n'aurait pas pu réaliser l'œuvre de législateur ${ }^{25}$.

23. Ibid., page 381

24. Pour mieux saisir la «nature» du législateur, tenir compte de ce passage du Second Discours: «... la commisération naturelle, qui, perdant de Société à Société presque toute la force qu'elle avoit d'homme à homme, ne réside plus que dans quelques grandes Âmes Cosmopolites, qui franchissent les barriéres imaginaires qui séparent les Peuples, et qui, à l'exemple de l'être souverain qui les a créés, embrassent cout le Genre-humain dans leur bienveillance (Pléiade III, page 178). " $\grave{A}$ remarquer que l'Être souverain a créé les peuples plutôt que les grandes âmes.

25. Par ailleurs, Rousseau s'est employé à deux reprises à imaginer des projets de constitution. Voir le Projet de constitution pour la Corse et les Considérations sur le 
Être au-delà de la nature humaine, au-delà de la cité, visant une œuvre contradictoire ou du moins difficile à concevoir, le législateur que Rousseau invente dans le Contrat social n'est au fond qu'une possibilité jamais vraiment actualisée : aussi l'auteur lui donne des dimensions mythiques plutôt que réelles ${ }^{26}$. Multipliant et accentuant les contradictions dans sa description de l'œuvre du législateur, Rousseau lui-même semble reconnaître que ce personnage ne peut exister que dans une rêverie idéalisante. «Ainsi l'on trouve à la fois dans l'ouvrage de la législation deux choses qui semblent incompatibles : une entreprise au dessus de la force humaine, et pour l'éxécuter, une autorité qui n'est rien... Pour qu'un peuple naissant put goûter les saines maximes de la politique et suivre les règles fondamentales de la raison d'État, il faudrait que l'effet put devenir la cause, que l'esprit social qui doit être l'ouvrage de l'institution présidât à l'institution même, et que les hommes fussent avant les loix ce qu'ils doivent devenir par elles ${ }^{27}$. „ On sent bien, d'ailleurs, que d'accorder trop de réalité à cet être surhumainement puissant nuierait pour autant à l'impact politique pratique qu'escompte Rousseau ${ }^{28}$. N'empêche que l'égalité civile

gouvernement de Pologne et sur sa réformation projettée dans le troisième tome de l'édition de la Pléiade. Le sort des “projets» rappellent la relation que Rousseau entretient avec la pédagogie pratique : sachant dire comment faire plutôt que sachant faire lui-même, il laisse faire à d'autres. "Hors d'état de remplir la tâche la plus utile, joserai du moins essayer de la plus aisée ; à l'éxemple de tant d'autres je ne mettrai point la main à l'œuvre mais à la plume, et au lieu de faire ce qu'il fait je m'efforcerai de le dire. »Émile, Pléiade IV, page 264. Voir aussi Premier Discours, Pléiade III, page 30 .

26. Même les figures historiques auxquelles l'auteur fait allusion de temps en temps ne peuvent être des exemples du législateur. À un Lycurgue que loue Rousseau, à un Platon, à tout autre cas de législateur idéal qu'on puisse signaler dans le passé, il manque la théorie du contrat social : dans l'optique de Rousseau, sans elle, peut-on créer une constitution qui respecte l'homme et ainsi une société légitime? sans elle, peut-on être le législateur dont il parle dans le Contrat Social?

27. Du contrat social, Pléiade III, page 383

28. Car le Contrat social n'est pas seulement un traité intemporel décrivant une fois pour toutes les conditions et limites nécessaires d'une société légitime ; c'est simultanément un pamphlet qui doit ébranler les monarchies européennes. Le citoyen de Genève dénonce tout autant qu'il énonce. Ainsi, dès le second chapitre du premier livre de son traité, Rousseau argumente contre la royauté de droit divin en démantelant les trois arguments traditionnels de ses défenseurs: l'analogie de la famille, l'analogie du pasteur, la filiation royale remontant à Adam, premier monarque. Il montre entre autres que l'analogie faite à partir du berger n'est acceptable qu'en supposant une différence de nature entre les hommes : les rois sont alors des demi-dieux, ou les peuples des troupeaux de bêtes. «Comme un pâtre est d'une nature supérieure à celle de son troupeau, les pasteurs d'hommes, qui sont leurs chefs, sont aussi d'une nature 
que fonde le pacte social et qui, à son tour, fonde la volonté générale suppose théoriquement l'existence de ce surhomme. En considérant ces deux conditions sine qua non de la cité juste selon Rousseau : l'unanimité politique et morale du peuple et la toutepuissance d'un guide national, on comprend qu'on ait pu l'accuser d'avoir été le père du totalitarisme moderne.

Mais dans le Contrat social, on s'échappe de l'encerclement homogène et homogénéisant de la volonté générale par une autre percée. Il faut d'abord rappeler qu'on greffe le citoyen sur l'homme dans l'état de nature et ce pour le transformer autant que possible. «Je suppose les hommes parvenus à ce point où les obstacles qui nuisent à leur conservation dans l'état de nature, l'emportent par leur résistance sur les forces que chaque individu peut employer pour se maintenir dans cet état. Alors cet état primitif ne peut plus subsister, et le genre humain périroit s'il ne changeoit sa manière d'être ${ }^{29}$. " Mais la nature par définition ne peut jamais être anéantie: non seulement revient-elle au galop, mais elle ne nous quitte jamais tout à fait ${ }^{30}$. C'est ainsi que le citoyen, malgré les exigences théoriques du concept de volonté générale, garde sa volonté d'individu comme une sorte de doublure de sa volonté de citoyen. Dans tout régime, même le plus parfait, même celui du

supérieure à celle de leurs peuples. Ainsi raisonnoit, au raport de Philon, l'Empereur Caligula ; concluant assez bien de cette analogie que les rois étoient des Dieux, ou que les peuples étoient des bêtes. 》 (Ibid., page 353.) Dans le même ordre d'idées, ce n'est pas un hasard si le Contrat social se termine avec une apologie quasi historique des institutions de la Rome républicaine: il n'y a rien de plus éloigné en esprit de la première Rome païenne que les monarchies chrétiennes contemporaines de Rousseau. Aussi la figure du législateur ne peut qu'affaiblir la critique que Rousseau propose des monarchies et de la hiérarchisation politique fixiste qu'elles supposent et entretiennent.

29. Ibid., page 360. - Cette phrase est comme un résumé du mouvement décrit dans la seconde partie du Second Discours: là, Rousseau tentait d'expliquer par quels mécanismes naturels et par quelles étapes incontournables l'homme dans l'état de nature est amené à se transfo-mer en homme socialisé, et mal socialisé. Le Contrat social est le complément obligatoire du Second Discours en autant qu' il montre ce qui idéalement aurait dû se passer lorsque l'homme sortit de l'état de nature. C'est pourquoi Rousseau peut affirmer que : "Tout ce qu'il y a de hardi dans le Contrat social étoit auparavant dans le Discours sur l'inégalité... 》L Les Confessions, Pléiade I, page 407 .

30. «Quel est ce but? C'est celui même de la nature : cela vient d'être prouvé. Puisque le concours des trois éducations est nécessaire à leur perfecrion, c'est sur celle à laquelle nous ne pouvons rien qu'il faut diriger les deux autres. » Émile, Pléiade IV, page 247. "Celle à laquelle nous ne pouvons rien ", c'est l'éducation que fournit la nature. Voir aussi les paragraphes qui suivent immédiatement. 
Contrat social, il y a un tiraillement continuel entre la volonté générale et les volontés pré-civiles ou plus exactement infraciviles ${ }^{31}$.

C'est d'ailleurs là la source du problème que pose l'existence même du Gouvernement : si les membres du gouvernement n'avait que leurs volontés comme membres du souverain, ou leurs volontés comme membres du gouvernement, il n'y aurait aucun danger, ou, en tout cas, peu de danger, que le pouvoir du gouvernement vise une cible autre que celle fixée par le souverain. Bien mieux : la nécessité d'un gouvernement, c'est-à-dire d'une magistrature, seraitelle aussi grande s'il n'y avait aucune tension entre l'homme comme individu et l'homme comme citoyen ${ }^{32}$ ? Or cette belle unité n'existe justement pas. «Nous pouvons distinguer dans la personne du magistrat trois volontés essenciellement différentes. Premièrement la volonté propre de l'individu, qui ne tend qu'à son avantage particulier ; secondement la volonté commune des magistrats, qui se rapporte uniquement à l'avantage du Prince, et qu'on peut appeller volonté de corps...; en troisième lieu, la volonté du peuple ou la volonté souveraine... ${ }^{33}$ » Aussi le gouvernement monarchique est le plus dangereux parce qu'il est le parfait exemple de l'identification du gouvernement avec une volonté particulière : «... sous un magistrat unique cette même volonté de corps n'est, comme je l'ai dit, qu'une volonté particulière ${ }^{34}$.»

31. Voir Du contrat social, Pléiade III, pages 373 et 375 , tirées du chapitre intitulé « Des bornes du pouvoir souverain. ». Le chapitre suivant, « Du droit de vie et de mort. » est évidemment lié à ce problème de la difficile relation entre l'individu comme citoyen et le même individu comme être naturel : la mise à mort ou le sacrifice de sa vie est le cas limite où se révèlent les limites justement des régimes et de l'adhésion d'un individu au tout politique. Il n'est pas sûr que Rousseau ait résolu ce problème à son entière satisfaction. C'est la thèse de cet article que les tensions de sa pensée, ou plus exactement la nature de l'homme telle qu'il la concevait, l'en empêchaient : son œuvre est, en quelque sorte, une démonstration scientifique et une confession autobiographique de l'impossibilité de vivre en société. Voir Émile, Pléiade IV, pages 845-846.

32. "Le Gouvernement reçoit du Souverain les ordres qu'il donne au peuple, et pour que l'État soit dans un bon équilibre il faut, tout compensé, qu'il y ait égalité entre le produit ou la puissance du Gouvernement pris en lui-même et le produit ou la puissance des citoyens, qui sont souverains d'un côté et sujets de l'autre. " Du contrat social, Pléiade III, pages 396 . Toute la description du gouvernement fait implicitement référence à la force: force exécutive, le gouvernement est essentiellement une «force réprimante ».

33. Ibid., pages $400-401$.

34. Ibid., page 402 . 
Enfin, c'est à la lumière de la rémanence de cette autonomie naturelle humaine qu'on comprendra que le contrat social puisse être résilié en tout moment et chacun des membres d'une cité rétabli instantanément dans ses pleins droits naturels ${ }^{35}$. Ailleurs, Rousseau conçoit même une existence entière où un homme voudrait « ... ni obéir ni commander... » et demeurerait « ... libre et indépendant, lui et sa postérité... » quoiqu'il jouirait des avantages de la vie en société, quoiqu'il vivrait au milieu d'hommes qui ont endossé le rôle de citoyens. Comme l'indique Rousseau un tel état d'exception exige un type humain spécial ${ }^{36}$.

Il existe donc dans la théorie rousseauiste de la vie politique un irréductible élément qui est la négation même de la socialisation, élément qui peut même légitimer une existence apolitique, si ce n'est purement et simplement asociale: cela s'ajoute à la figure olympienne du législateur; cela la corrige. L' «au-delà» de la société est compensé par un « en deçà ». Le Contrat social fait donc la preuve de l'existence chez Rousseau de deux types humains qui se trouvent aux marges de la société civile - mais à des marges opposées ou plus exactement inégales. Cette structure se retrouve ailleurs dans son œuvre.

Il faudrait sans doute réfléchir sur le statut relatif de ces trois types humains aux yeux de Rousseau: s'il faut choisir, et il faut toujours choisir d'une façon ou de l'autre, lequel des trois hommes

35. «Les clauses de ce contrat sont tellement déterminées par la nature de l'acte, que la moindre modification les rendroit vaines et de nul effet... le pacte social étant violé, chacun entre alors dans ses premiers droits et (reprend) sa liberté naturelle, en perdant la liberté conventionnelle pour laquelle il y renonça. » Ibid., page 360. - Voir aussi sur le droit naturel de tuer le tyran, Second Discours, Pléiade III, page 191 ; et sur le droit de l'individu à « renoncer au contrat par lequel il tient à la communauté... », Émile, Pléiade IV, page 833.

36. Voir le Second Discours, Pléiade III, page 195. C'est la fameuse première note qui traite du cas du Perse Otanès : il n'est ni magistrat, ni à proprement parler citoyen, pourtant il demeure dans la cité avec les siens. Il est important de rattacher cette note de Rousseau avec le texte qu'elle complète, à savoir la lettre dédicatoire du Second Discours, laquelle décrit en résumé la cité du Contrat social: Rousseau pense celui qui s'exclut de la société au moment même de résumer les conditions d'existence d'une société idéale. - Ajoutons qu'après être arrivé aux principales conclusions que propose le Contrat social, Émile veut être un homme semblable à Otanès, à savoir un être apolitique (Émile, Pléiade V, pages 855-857); son pédagogue n'est pas d'avis qu'une telle existence soit la meilleure pour Émile (pages 858-960). Ce qui revient à dire qu'Émile est finalement un homme qui a reçu une éducation extraordinaire, mais qui n'en demeure pas moins assez ordinaire. 
est le plus «homme»? Notons en attendant de trouver une réponse à cette question une des ironies de la vie de Rousseau: après s'être imaginé, en écrivant le Contrat social, le législateur des législateurs, après s'être hissé jusqu'à un au-delà de la société dont il décrit les propriétés et les mécanismes, le rejet presque universel de son traité l'a obligé d'incarner l'individu en-deçà de la société, le promeneur solitaire errant sans patrie ni pays d'adoption ${ }^{37}$.

\section{Émile, son maître, Rousseau}

Si le Contrat social fut pour Rousseau source de difficultés personnelles considérables, elles ne firent que s'ajouter à celles que lui procura l'Émile. De fait, ces livres ont eu l'honneur d'être condamnés par les autorités françaises et genevoises, puis brûlés sur la place publique. Pour expliquer ces accueils semblablement intransigeants, il ne suffit pas de souligner que l'Émile reprend dans son cinquième livre l'essentiel des thèses du Contrat social. Car l'œuvre pédagogique de Rousseau mérita la rage publique à son propre titre. En effet l'Émile, qui porte comme sous-titre $D e$ l'éducation, s'attaquait systématiquement à toutes les opinions communes de son temps en matière d'éducation et de religion; plus particulièrement, l'auteur y critiquait les collèges religieux français ${ }^{38}$. L'éducation et la politique, thèmes jumeaux qui intéressent

37. Ajoutons, en guise de confirmation, que son sage idéal plus qu'humain existe plus souvent qu'autrement selon les sentiments et les rythmes de l'inscience, de la solitude et de la liberté plutôt que selon les raisons et les stratagèmes de la sagesse, de l'engagement et de la manipulation. A cer effer, il est utile de rappeler la charmante scène de la cinquième rêverie où Rousseau établit une colonie de lapins. Il ressemble au législateur du Contrat social: "Je donnai cette idée au Receveur qui fit venir de Neufchatel des Lapins males et femelles et nous allames en grande pompe, sa femme, une de ses sceurs, Therese et moi, les établir dans la petite Isle, où ils commençoient à peupler avant mon depart et où ils auront prospéré sans doute s'ils ont pu soutenir la rigueur des hivers. La fondation de cette petite Colonie fut une fete (Les Rêveries $d u$ promeneur solitaire, Pléiade I, page 1044 - mais aussi Les Confessions, Pléiade, page 644.). »Voir surtout la suite qui est la fameuse description d'une rêverie où le législateur-fondateur ne pense plus du tout à sa colonie. Cet exemple parodique du sage législateur se sépare de son œuvre pour rêver paisiblement et retrouver, selon un mode supérieur sans doute, la merveilleuse indolence de l'homme dans l'état de nature. Voilà le législateur-rêveur qu'est Jean-Jacques.

38. Il ne fait aucun doute que l'hétérodoxie de la profession de foi du Vicaire Savoyard fut pour beaucoup dans les condamnations tant protestantes que catholiques. - Pour les collèges, voir, par exemple, Émile, Pléiade IV, page 250. Mais cette critique est reprise constamment un peu partout dans le traité. 
tous les philosophes depuis Socrate, seront toujours des sujets qui réveilleront les hommes même les plus insensibles aux questions philosophiques et où l'hétérodoxie provoquera l'indignation la plus vive ${ }^{39}$.

L'objectif poursuivi dans l'Émile est de montrer comment éduquer ou former un homme naturel. Il ne faut pas confondre cette expression, "homme naturel ", avec une autre du vocabulaire rousseauiste qui lui ressemble assez: "homme dans l'état de nature». L'homme dans l'état de nature est un être humain qui dépasse à peine l'état d'animalité. À l'opposé, l'homme naturel, Émile, n'est pas voué à vivre en sauvage solitaire au fond des forêts, ni même en illettré balourd dans une société sous-développée ; au contraire, l'idéal que Rousseau vise pour lui est plus près de l'homme «autarcique » des Grecs : un homme pour tous les temps et pour toutes les sociétés. "Il y a bien de la différence entre l'homme naturel vivant dans l'état de nature, et l'homme naturel vivant dans l'état de société. Émile n'est pas un sauvage à releguer dans les déserts ; c'est un sauvage fait pour habiter les villes. Il faut qu'il sache y trouver son nécessaire, tirer parti de leurs habitants, et vivre, sinon comme eux, du moins avec eux ${ }^{40}$.» «Vivre avec» n'est pas "vivre comme»: Émile sera un homme pleinement développé : sensible, rationnel, civilisé, fait pour vivre dans une société, même s'il n'en fera pas vraiment partie, même s'il sentira et entretiendra toujours une distance morale entre eux et lui. Par ailleurs, il est permis de se demander si l'Émile ne se solde pas, selon les visées même de l'auteur et d'après les indications qu'il

39. «Lisez la République de Platon. Ce n'est point un ouvrage de politique, comme le pensent ceux qui ne jugent des livres que par leurs titres. C'est le plus beau traité d'éducation qu'on ait jamais fait." Ibid., page 250. Si la République porte sur l'éducation, l'Émile peut bien porter aussi sur la politique, contrairement à ce qu'indique son sous-titre: les deux chefs-d'œuvre philosophiques se répondent, en abordant des thèmes éternellement liés.

40. Ibid., pages 483-484. - Émile serait une espèce d'homme civilisé qui n'endosse pas la manière d'être du citoyen. On aurait raison de le croire et quant à l'objectif qui est proposé au début du livre et même quant au résultat «effectif » obtenu à la fin. Pourtant, Émile est l'équivalent du citoyen du Contrat social, au moins en autant qu'il est dépassé par la figure du gouverneur et qu'il dépasse lui-même celle de l'homme vivant aux marges de la société. Peut-être en dernière analyse faut-il voir en Émile une espèce de compromis entre le citoyen et l'homme solitaire. Voir la note et le texte suivants. - Pour cette définition de l'homme « autarcique », voir la Vie d'Alcibiade de Plutarque 
nous laisse, par un échec partiel. Cette possibilité se révèle au lecteur à mesure que Sophie apparaît dans le traité : être envoûté par l'incarnation de la fernme idéalisée, épouser Sophie, lui faire des enfants, vivre aux côtés de Sophie, qui en tant que femme vit soumise à l'opinion, n'est-ce pas rater, en partie du moins, l'objectif de la totalité qui devait commander toute l'éducation d'Émile ${ }^{41}$ ? Mais du même coup Émile incarne au moins partiellement la figure de l'homme normal, intégré à sa société, liée par tout ce qu'il a de plus cher aux hommes qui l'entourent. Si Émile est exceptionnel, c'est comme le citoyen du Contrat social qui est hors de l'ordinaire.

La figure toute imaginaire d'un Émile à éduquer est accompagnée, comme il se doit, par celle de son pédagogue. Dès les premières pages de son traité, Rousseau souligne le caractère exceptionnel de l'homme chargé de la formation de l'être hors pair qu'est Émile. Il l'appelle un «rare mortel» et un "prodige». Mesure non-négligeable de la hauteur de la tâche: l'auteur luimême la refuse parce que trop difficile. "Un gouverneur! quelle ame sublime... en vérité, pour faire un homme, il faut être pére ou plus qu'homme soi-même. » On remarquera sans difficulté que le gouverneur, tel que décrit par Rousseau, ressemble à s'y méprendre au législateur du Contrat social. Et ce jusqu'à posséder lui aussi des qualités contradictoires et donc incompatibles. «Je voudrois qu'il fut lui-même enfant s'il étoit possible, qu'il put devenir le compagnon de son élêve, et s'attirer sa confiance en partageant ses amusements ${ }^{42}$. Par ailleurs, ce compagnon de l'élève a un contrôle

41. Si Émile doit être libre, il est, disons, regrettable qu'il soit l'esclave d'une Sophie, ellemême esclave de l'opinion. - Sur la question du lien qui retient Émile à partir de Sophie, voir, par exemple, Ibid., pages $639 ; 789-790 ; 796 ; 799 ; 816-818 ; 823 ; 833$; $853 ; 857-860 ; 865$ : Émile ne peut plus se satisfaire d'être bon parce qu'il n'est plus vraiment indépendant; la liberté morale que chante si bien Rousseau est, pour le moins, difficile à réconcilier avec ses conceptions fondamentales de l'unité, de la simplicité et de la bonté humaines. - D'ailleurs, la suite de l'Émile, Les Solitaires, que Rousseau a projerée, mais n'a jamais rerminée, nous montre dès les premières pages un Émile ravagé par l'infidélité de son épouse. La solution que découvre Émile, toute rousseauiste, est de se séparer de l'autre, de la famille, et ainsi de la patrie et même de la chrétienté. On pourrait dire que l'éducation d'Émile n'est réussie ou complétée, qu'elle ne fait ses preuves, qu'après l'épreuve de l'infidélité de Sophie ; mais alors c'est que cette éducation le préparait à vivre tout à fait hors des cadres de la famille et de la société : sur une île, loin de la civilisation européenne, sans enfant. Voir Pléiade IV, pages cliii à clxviii et pages 879-924, particulièrement 905-912. On retrouvera plus loin un exemple de cette «chute» de la vie socialisée à la solitude en examinant le cas de Julie dans la Nouvelle Héloise.

42. Émile, Pléiade IV, pages 263-265. 
parfait de ses propres sentiments, de ses opinions et de ses moyens d'action. En somme, pour éduquer Émile comme Rousseau le voudrait, il faudrait un enfant qui soit déjà un adulte.

De plus, à la limite, le pédagogue doit contrôler non seulement Émile, mais aussi tout ce qu'Émile voit et tous ceux qu'il rencontre. C'est ainsi qu'au début du premier livre de son traité, Rousseau se moque de la faiblesse humaine en matière d'éducation. «Or de ces trois éducations différentes, celle de la nature ne dépend point de nous ; celle des choses n'en dépend qu'à certains égards ; celle des hommes est la seule dont nous soyons vraiment maitres; encore ne le sommes-nous que par supposition: car qui est-ce qui peut espérer de diriger entièrement les discours et les actions de tous ceux qui environnent un enfant ${ }^{43}$ ? "Pourtant, comme le montre une lecture attentive du traité, ce contrôle des faits et gestes de chacun, ce contrôle des plus petits événements de la vie d'Émile font partie des pouvoirs du pédagogue que Rousseau suppose dans l'Émile. Rappelons à titre d'exemple le conflit avec Robert le jardinier et celui avec le joueur de gobelets ${ }^{44}$, où une savante mise en scène, incluant les réactions verbales et émotives des protagonistes, préside aux deux rencontres et permet au pédagogue d'éduquer par les choses, plutôt que par les mots. C'est en poursuivant la lecture de l'Émile qu'on se rend vraiment compte de l'énormité de la tâche du pédagogue d'Émile. Tâche quasi divine en ceci qu'il connaît, ou devrait connaître, parfaitement les pensées les plus intimes de son protégé, qu'il prévoit sans faute ses moindres réactions, qu'il sait infailliblement parer à toutes ses feintes et affronter toutes les difficultés. Le premier, mais certes pas le dernier, exemple de ce devoir inhumainement difficile est la lecture des pleurs : à partir d'un signe aussi indistinct que les cris et les larmes d'un poupon le pédagogue doit deviner les premiers mouvements d'une âme encore inconsciente d'elle-même et parer aux dangers d'une mauvaise éducation qui menace depuis le tout début de faire avorter le processus ${ }^{45}$. Conçue de cette façon, la

43. Ibid., page 247. - L'italicisation n'est pas dans l'original.

44. Ibid., pages 330-332; pages 437-440. - Toutes les circonstances sont suprêmement importantes. $\AA$ preuve : «Un éclat de rire indiscret peut gâter le travail de six mois et faire un tort irréparable pour toute la vie (page 328).» - Voir aussi pages 801-802 : le hasard n'existe pas puisque le gouverneur remplace la Providence.

45. Émile, Pléiade IV, pages 286-292. - Voir aussi pages 510-511 et les premières rencontres entre Émile et Sophie, où le pédagogue lit dans leurs cœeurs comme dans un 
charge du pédagogue est écrasante; pourtant Rousseau la double au livre cinquième lorsqu'il apprend à son lecteur que tout en contrôlant jusque dans ses moindres détails l'éducation d'Émile, le gouverneur surveillait comme de loin, et depuis le débur, celle de Sophie ${ }^{46}$.

Nulle part la douce tyrannie du précepteur n'apparaît-elle plus évidemment que lorsque la sexualité d'Émile se manifeste. A partir de ce moment, la relation entre le maître et l'enfant se modifie radicalement. Certes, depuis le début, le pédagogue est appelé à contrôler, dans les deux sens du mot, toutes les réactions d'Émile. Mais la force de la pulsion sexuelle et le péril où elle met l'œuvre pédagogique sont tels qu'il faut changer de méthode lorsque Émile atteint la pleine adolescence. Il s'agit d'abord d'initier le jeune homme à l'idée de Dieu et simultanément à celle des devoirs moraux : le pédagogue est donc conduit, comme le législateur a vec les citoyens, à utiliser l'influence civilisatrice de la religion, ou plus exactement dans ce cas-ci l'impact moral d'une profession de foi ; après avoir retenu le développement intellectuel d'Émile, il s'agit de l'accélérer énormément, sans doute plus vite que ne le

livre. Les parties romanesques de l'Émile sont troublantes en autant que toutes les répliques et les réactions paraissent être prévues et commandées par le pédagogue ; il surveille, il épie, il agit sans qu'on s'en rende compte, il domine la scène qu'il provoque, examine et rapporte : sa clairvoyance et son efficacité paraissent alors moins divines que diaboliques, moins providentielles que tyranniques.

46. Autre élément troublant de l'Émile : la façon dont Rousseau, après avoir chanté les gloires de la paternité et de la maternité responsables, éloigne d'un tour de main les parents d'Émile: «Émile est orphelin. Il n'importe qu'il ait son pére et sa mére.» (Émile, Pléiade IV, page 267 ; voir aussi la page 765 .) Â la limire, la mère et le père ne sont tolérés que comme des instruments de l'art et, il faut le reconnaître, de la sollicitude du gouverneur. Par contre, à la toute fin du traité, Émile annonce que Sophie et lui-même prendront soin de l'éducation de leur enfant. Pour bien éduquer, faut-il des parents et l'atmosphère d'une famille ou non? Ce flottement dans le texte de Rousseau quant aux responsables de l'éducation de l'enfant répond à d'autres flottements, déjà signalés, portant sur le sens même de l'éducation : Émile est fait pour pouvoir vivre seul et pourtant il ne vit qu'avec les autres, même plus il a besoin des autres, du moins a-t-il besoin de Sophie; Émile est fait pour être libre, et pourtant on n'a jamais, avant Rousseau, imaginé une éducation plus totalitaire que la sienne. Que l'on compare ces deux citations : « Forcé de combattre la nature ou les institutions sociales, il faut opter entre faire un homme ou un citoyen; car on ne peut faire à la fois l'un et l'autre (page 248). » et « Un pére, quand il engendre et nourrit des enfans ne fait en cela que le tiers de sa tâche. Il doit des hommes à son espéce, il doit à la societé des hommes sociables, il doit des citoyens à l'État (page 262). "S'agit-il d'éduquer un individu ou un citoyen, ou l'un et l'autre ? C'est là l'ambiguité de fond de l'Émile et, il faut le dire, de toute la pensée de Rousseau. 
permet sa compréhension effective : la foi remplace ici l'expérience. "En développant le naturel, nous avons donné le change à sa sensibilité naissante; nous l'avons réglée en cultivant la raison. Les objets intellectuels moderoient l'impression des objets sensibles. En remontant au principe des choses, nous l'avons soustrait à l'empire des sens ; il était simple de l'élever de l'étude de la nature à la recherche de son auteur ${ }^{47}$. " Ensuite, deuxième stratégie, il faut occuper Émile par l'image d'une femme, image faite pour tromper ses pulsions sexuelles naissantes; et en même temps il faut lui faire plier l'échine au nom de la sollicitude clairvoyante de l'amipédagogue. Il s'agit d'aliéner Émile de lui-même de façon que le gouverneur le domine parfaitement. "Il $\mathrm{m}$ 'a fallu quinze ans de soins pour me ménager cette prise. Je ne l'élevois pas alors, je le préparois pour être élevé; il l'est maintenant assés pour être docile; il reconnoit la voix de l'amitié et il sait obéir à la raison. Je lui laisse, il est vrai, l'apparence de l'indépendance, mais jamais il ne me fut mieux assujeti, car il l'est parce qu'il veut l'être. Tant que je n'ai pu me rendre maitre de sa volonté je le suis demeuré de sa persone; je ne le quittois pas d'un pas. Maintenant je le laisse quelquefois à lui-même parce que je le gouverne toujours ${ }^{48}$, $) \mathrm{Ce}$ début " prometteur » se poursuit tout au long de l'idylle amoureux d'Émile et de Sophie : le pédagogue s'immisce en tiers partout et toujours dans leurs rencontres et leurs conversations. Un envahissement qui mène le pédagogue jusque dans le lit conjugal du jeune couple : il se mêle de légiférer sur la fréquence de leurs rapports sexuels ${ }^{49}$. Le labeur pédagogique frise le totalitarisme. Pour en revenir au gouverneur, le pédagogue qu'imagine Rousseau est un surhomme, il existe au-delà des limites de la condition humaine : il est un homme naturel qui n'a pas eu besoin de la pédagogie qu'il

47. Émile, Pléiade IV, page 636. Malgré I'affirmation de Rousseau quant à la simplicité de cette tâche, il est patent par les passages qui précèdent la profession de foi (pages 550-557) que ces considérations métaphysiques ne seront pas reçues par Émile suite à une réfléxion proprement philosophique qui l'aurait conduit à une évidence quelqconque : la profession de foi est reçue comme il se doit sur le mode de la foi.

48. Ibid., page 661. - Pour ce qui est de l'utilisation de l'imaginaire comme leurre, voir les pages 656-657. - Cette tendance manipulatrice de l'œuvre du pédagogue apparaissait bien avant, par exemple au tout premier livre à la page 274 : le gouverneur, idéalement, serait tout seul pour mieux contrôler et dominer Émile. En dernière analyse, Rousseau, chantre de la transparence et de la liberté, serait le créateur d'une des figures les plus dominatrices et les plus opaques de la littérature : I'éducateur d'Émile.

49. Ibid., pages 861-867. 
utilise avec Émile, qui s'est donc éduqué par lui-même et qui retourne pour ainsi dire auprès des hommes pour faire du bien à l'un d'eux; mais ce bien exige un labeur de titan.

Par contre, selon la suite de l'Émile, intitulé Les Solitaires, mais contrairement à un des principes premiers du système pédagogique ${ }^{50}$, le gouverneur s'échappe en quelque sorte de ses épuisantes responsabilités une fois terminé son ouvrage. Échappée qui est malheureuse du point de vue d'Émile, mais qui révèle encore une fois cette tendance de Rousseau à chercher et trouver le bonheur ailleurs que dans l'action de l'honnête homme, et même ailleurs que dans la transcendance quasi divine : la retraite dans un asile inconnu et la séparation d'avec les hommes semblent être les meilleures conditions du bonheur personnel du pédagogue ${ }^{51}$. Cette échappée ultime du gouverneur nous ramènera à certains aveux faits par Rousseau à la fin du livre quatrième : il y décrit une vie « idéale », qui serait la sienne s'il devenait soudainement riche. Rien de plus banal qu'une telle rêverie, croirait-on. Elle prend une importance certaine en raison du contexte: le mode de vie de Rousseau serait bien différent de tout ce qui est proposé jusque-là dans l'Émile.

De quoi est faite l'existence rêvée de Jean-Jacques ${ }^{52}$ ? De loisir, d'abord. Non pas que Rousseau désire vivre dans l'inaction : ses journées seraient remplies de randonnées avec des amis ou d'occupations les plus ordinaires, mais allégées de leur poids de

50. Ibid., page 267-268.

51. "Étoit-ce par vous, pére cruel, que devoit commencer ce déclin? Par quelle fatalité putes-vous quiter cette vie paisible que nous menions ensemble, comment mes empressémens vous rebutérent-ils de moi? Vous vous complaisiez dans vôtre ouvrage ; je le voyois, je le sentois, j'en étois sur. Vous paroissiez heureux de mon bonheur; les tendres caresses de Sophie sembloient flater vôtre cœur paternel; vous nous aimiez, vous vous plaisiez avec nous, et vous nous quitâtes! Sans vôtre retraite je serois heureux encore; mon fils vivroit peut-être, ou d'autres mains n'auroient point fermé ses yeux. Sa mére, vertueuse et chérie vivroit elle-même dans les bras de son époux. Retraite funeste, qui m'a livré sans retour aux horreurs de mon sort! Non, jamais sous vos yeux le crime et ses peines n'eussent approché de ma famille; en l'abandonant vous m'avez fait plus de maux que vous ne $m$ 'aviez fait de biens en toute ma vie. " Les Solitaires, Pléiade IV, page 884. - Sans doute faut-il reconnaître dans ces reproches l'excès de l'émotion du moment. Mais n'y a-t-il beaucoup de vrai dans ce que dit ici Émile? Au moins ceci : il met le lecteur face au problème du retrait du pédagogue et du sens de ce retrait.

52. Pour tout ce paragraphe, voir le dérail de la descriprion de Rousseau dans l'Émile, Pléiade IV, pages 678-691. 
sérieux. Les activités quotidiennes sont transformées en jeux insouciants. De voyages, ensuite. Car Rousseau ne s'en tiendrait pas à un seul pays: il passerait l'été à Naples et l'hiver à SaintPétersbourg; où il trouverait le plaisir, là se trouverait sa patrie, nous dit-il. On admettra que ce n'est là ni la devise d'un sage ni celle d'un citoyen. De femmes libres, enfin. Certes il n'est pas question d'entretenir de jeunes dames pour satisfaire à toute heure les phantasmes libidineux d'un vieil homme : Rousseau est conscient du ridicule d'un tel comportement; mais il n'est pas insensible à la beauté, il a besoin de supports indépendants et égaux sur lesquels répandre les effets de son activité bienveillante mais elle-même indépendante. En somme, après avoir décrit le sérieux de la tâche du gouverneur, après avoir «rapporté » la profession de foi du Vicaire Savoyard ${ }^{53}$, après avoir décrit les efforts d'Émile pour trouver sa Sophie, Rousseau brosse un tableau où l'épouse, la religion et le labeur sont étrangement absents. Silence combien éloquent : encore une fois, on retombe dans une vie qui est bien endeçà des exigences de la socialisation et de la digne sagesse. Ce ne sera pas le dernier exemple de cette chute. La question finale serait de savoir si la «chute» n'est pas, dans l'optique de Rousseau, une reconquête de l'état de nature, mais selon un mode nouveau et supérieur : la chute est délivrance et affranchissement.

\section{Trois membres d'une famille}

Les malheurs qui poursuivirent Rousseau à partir de la publication du Contrat social et de l'Émile ne doivent pas conduire à conclure que le citoyen de Genève ne connut que des déboires

53. Il serait impossible en cette occasion de faire des remarques adéquates sur le profession de foi du Vicaire Savoyard, même en se limitant strictement à la question de sa relation à la triade qui est analysée ici. Signalons au moins que ce texte crucial de l'œuvre de Rousseau paraît problématique pour au moins deux raisons: sa pasition dans le processus pédagogique, alors que d'un coup Émile, ou plus exactement son équvalent. est confronté sans préparation intellectuelle véritable aux problèmes les plus abstrus de la métaphysique ; et ensuite l'apparition soudaine dans la profession de foi d'une conception résolument dualiste de l'être humain. Quant à ce dernier point : comparer les pages 522 et 523 (surtout la longue note) et "Si la conscience est l'ouvrage des préjugés, j'ai tort, sans doute, et il n'y a point de morale démontrée ; mais si se préférer à tout est un penchant naturel à l'homme, et si pourtant le prémier sentiment de la justice est inné dans le couur humain, que celui qui fait de l'homme un être simple léve ces contradictions, et ie ne reconnois qu'une substance (page 584). 
durant sa carrière littéraire. Certes, il voudrait faire croire que sa vie d'écrivain ne fut qu'un long, très malheureux et presque involontaire accident de parcours; le bon Jean-Jacques n'aurait voulu être rien de plus qu'un honnête citoyen de Genève; puni pour sa faiblesse et sa témérité, rejeté de tous et poursuivi par les forces politiques et culturelles conjuguées, il se replia dans une envoûtante solitude, qui était sa première assiette morale. C'est l'image qu'il voudrait nous laisser en écrivant Les Confessions et ses autres œuvres biographiques ${ }^{54}$. Mais en rester là serait à la fois une inexactitude historique et une occasion de perdre de vue la pensée de Rousseau déjà de son vivant. Nous ne ferons que signaler ici le succès originel éclatant du Premier Discours, par exemple, ou, dans un tout autre genre, la réussite brillante du Devin du village, pour nous tourner vers la Nouvelle Héloïse qui remporta un véritable triomphe. Rousseau lui-ınême, quoique assez intéressé à se montrer le paria des Parisiens, ne peut s'empêcher de souligner cette réception extraordinaire au point d'exprimer à cette occasion des sentiments peu philosophiques. "Qoique la Julie qui depuis longtems était sous presse ne parut point encore à la fin de 1760 , elle commençoit à faire grand bruit... Tout Paris était dans limpatience de voir ce roman; les librairies de la rue St. Jacques et celui du Palais royal étoient assiegés de gens qui en demandoient des nouvelles. Il parut enfiı, et son succés, contre l'ordinaire, répondit à l'empressement avec lequel il avoit été attendu... dans le monde il n'y eut qu'un avis, et les femmes surtout s'enivrérent et du Livre et de l'auteur au point qu'il y en avoit peu, même dans les hauts rangs, dont je n'eusse fait la conquête si je l'avors entrepris ${ }^{55}$.» D'ailleurs, Rousseau avait déià utilisé la

54. Quand il est question de Rousseau, le penseur nous conduit toujours à l'homme et l'homme au penseur : Rousseau est juge de Jean-Jacques et Jean-Jacques modèle pour Rousseau. En somme, il y a une parfaite «continuité entre les écrits doctrinaux de Rousseau et ses écrits intimes; voilà ce qui autorise — plus même : oblige — ̀̀ se tourner vers ses œuvres autobiographiques quand on veut connaitre mieux l'une des voies de l'homme qu'il a'tracées, celle de l'individu solitaire. » Tzvetan I odorov, Frêle bonheur: Essai sur Rousseau, Hachette, 1985, pages 66-67. - Sur le necessaire passage par la biographie dans une interprétation complète de la pensée de Rousseau, voir Jean STAROBINSKI, Jean-Jacques Rousseau: La transparence et l'obstacle, NRF, Éditions Gallimard, 1971, page 6. Il serait donc du plus haut intérêt d'examiner le mieux connu des écrits biographiques, Les Confessions, dans l'espoir de trouver une espèce de contrepreuve de l'hypothèse des figures en trio exposée jusqu'ici à partir des auvres « objectives» ou doctrinales de Rousseau.

55. Les Confessions, Pléiade I, page 545. 
Nouvelle Héloïse dans une opération de séduction menée auprès de Madame la Maréchale de Luxembourg: trop gêné pour tenter d'amuser cette grande dame par ses reparties rares et peu spirituelles, il imagina l'expédient de lire son roman à celle qui allait se montrer une amie bien utile dans les temps difficiles à venir ${ }^{56}$. Un tel succès porterait certains à croire que la Nouvelle Hélö̈se, même de l'avis de l'auteur, n'ait que peu à faire avec la "vraie» pensée de Rousseau, le sérieux étant inalliable avec le léger, la vertu avec la passion, la vérité avec l'amour.

Pourtant malgré la différence remarquable entre les réceptions successives des deux plus volumineux textes que Rousseau ait publiés, malgré des différences importantes d'atmosphère et de thèmes, il y a une filiation très certaine entre l'Émile et la Nouvelle Héloïse. C'est Rousseau qui l'affirme dans ses Confessions : «Depuis lors La nouvelle Héloïse parut encore avec la même facilité, j'ose dire avec le même applaudissement, et, ce qui semble presque incroyable, la profession de foi de cette même Héloïse mourante est exactement la même que celle du Vicaire Savoyard... tout ce qu'il y a de hardi dans l'Émile étoit auparavant dans la Julie ${ }^{57}$. " Mais le roman offre une preuve encore plus impressionnante de cette liaison en autant que celle-là est justement tirée à la fois de la doctrine de Rousseau et de l'essentiel de la trame de son roman : si Monsieur de Wolmar accepte de recevoir l'amant de son épouse au sein même de sa famille, situation délicate, voire scabreuse, et, comme le montrent certains épisodes bien marqués du roman,

56. «Le sucçés de cet expédient passa mon attente. Made de Luxembourg s'engoua de la Julie et de son Auteur ; elle ne parloit que de moi, ne s'occupoit que de moi, me disoit des douceurs toute la journée, m'embrassoit dix fois le jour. Elle voulut que j'eusse toujours ma place à table à côté d'elle, et quand quelques Seigneurs vouloient prendre certe place, elle leur disoit que c'étoit la mienne et les faisoit mettre ailleurs. 》 Ibid., pages 522-523. On notera que l'engouement de Madame la Maréchale préfigure celle des grandes dames et du Tout-Paris (voir le texte principal). Des aveux assez surprenants de la part de celui qui proteste dans les mêmes pages: "Car depuis quelque tems, je formois le projet de quitter tout à fait la litrérature et surtout le métier d'Auteur. Tout ce qui venoit de m'arriver m'avoit absolument dégouté des gens de lettres, et j’avois éprouvé qu'il étoit impossible de courir la même carriére sans avoir quelques liaisons avec eux. Je ne l'étois guéres moins de gens du monde et en général de la vie mixte que je venois de mener, moitié à moi-même, et moitié à des sociétés pour lesquelles je n'étois point fait (page 514). » Rousseau, l'homme veut-il donc vivre pour lui ou par rapport aux autres? Mais nous n'en sommes pas à une ambiguïté près avec Rousseau.

57. Ibid., page 407. 
grosse de dangers pour tous les protagonistes, si Monsieur de Wolmar attire même Saint-Preux auprès de Julie et les laisse souvent seuls ensemble, c'est qu'il est question pour lui d'assurer la saine éducation de ses enfants : Saint-Preux sera le gouverneur des enfants Wolmar. De plus, les principes pédagogiques de Monsieur de Wolmar et, conséquemment de son épouse, sont ceux que propose et exemplifie Rousseau dans l'Émile ${ }^{58}$. C'est comme si le traité d'éducation de Rousseau expose en long et en large l'œuvre que Julie avait commencé de réaliser et que Saint-Preux aurait mené à terme. Inversement, la seconde partie de la Nouvelle Hélö̈se étale au grand jour l'œuvre d'un père cherchant un gouverneur qui sache le seconder et le remplacer, œuvre de séduction qui dans l'Émile était laissée dans le vague ${ }^{59}$. Abordons donc le grand roman dans l'espoir de pouvoir éclairer à nouveau le trio d'archétypes qui fait l'objet de cette recherche ${ }^{60}$.

Le sujet de la Nouvelle Héloise est l'amour, ou plus exactement deux sortes d'amour : l'amour modéré, fondement de la société, et l'amour-passion, dynamite de cette même société. Il n'y a aucun doute que la figure dominante du grand roman de Rousseau est

58. Voir Julie ou la Nowvelle Héloìse, Cinquième partie, Lettre III, Pléiade II, pages 557-586.

59. "Qui donc élévera mon enfant? Je te l'ai déjà dit, toi-même. Je ne le peux. Tu ne le peux!... fais-toi donc un ami. Je ne vois point d'autre ressource. " Émile, Pléiade IV, page 263. Voir aussi la page 276. Il est remarquable, parce que tout à fait conforme à cette exhortation, que la lettre sur l'éducation de la Nouvelle Héloïse est précédée par la peinture d'une scène de bonheur familial, dans laquelle Saint-Preux se trouve inclus en tant qu'ami du couple. Julie ou la Nouvelle Hélouse, Pléiade II, pages 557-561. Tout a une forte teneur autobiographique chez Rousseau. Ne se décrit-il pas comme suit : "Je lui trouve aujourdui les traits du Mentor d'Émile. Peut-être dans sa jeunesse lui aurois-je trouvé ceux de St. Preux. "Rousseau juge de Jean Jaques, Pléiade I, page 778. Ce qui est une autre façon d'affirmer l'unité foncière des deux ceuvres : à travers l'unité dans sa personne de deux personnages clés.

60. On pourrait objecter non sans raison que les protagonistes de la Nouvelle Héloüse forment un quintette et qu'en conséquence appliquer comme on le fait ici le schème du trio, c'est se vouer à déformer l'œuvre. À quoi il faut répondre qu'il ne s'agit pas ici d'interpréter de façon exhaustive le roman de Rousseau, mais de réfléchir sur une constante de sa pensée. Par ailleurs, il serait assez facile de souligner que Milord Édouard, par un choix délibéré de l'auteur, est finalement exclu du groupe des belles àmes que forme les quatre autres. Reste Claire. Malgré l'importance de son rôle dans le développement de l'intrigue amoureuse et le fait qu'elle est en quelque sorte promise à Saint-Preux, elle paraît vouée à une position secondaire particulièrement dans la seconde partic du roman. Ou pour le dire autrement: Wolmar et Claire se complètrent de façon que l'apparition du mari de Julie réduit d'autant le rôle de son amie. 
Julie. Cela est vrai non seulement pour le lecteur, observateur situé à l'extérieur de l'intrigue, mais encore pour les personnages du roman pris dans les fils de la trame. «N'as-tu jamais remarqué, mon Ange, à quel point tout ce qui t'approche s'attache à toi ? Qu'un pere et une mere chérissent une fille unique, il n'y a pas, je le sais, dequoi s'en fort étonner ; qu'un jeune homme ardent s'enflame pour un objet aimable, cela n'est pas plus extraordinaire ; mais qu'à l'age mur un homme aussi froid que $M$. de Wolmar s'attendrisse en te voyant, pour la première fois de sa vie; que toute une famille t'idolatre unanimement; que tu sois chere à mon pere, cet homme si peu sensible, autant et plus, peut-être, que ses propres enfans : que les amis, les connoissances, les domestiques, les voisins et toute une ville entiere, t'adorent de concert et prennent à toi le plus tendre intérêt : Voila, ma chere, un concours moins vraisemblable, et qui n'auroit point lieu s'il n'avoit en ta personne quelque cause particuliere ${ }^{61}$. » De plus, puisqu'il est question d'un roman et donc d'une intrigue et du développement psychologique de divers personnages, soulignons que l'évolution de Julie est la matière première de l'œuvre de Rousseau; Julie est le seul personnage qui change vraiment au cours du roman : Saint-Preux est amoureux de Julie depuis la première page, Wolmar est, comme il se doit, toujours égal à lui-même, Claire ne change en rien qu'elle soit fille, femme ou veuve; Milord Édouard, figure fantômatique, ne se révèle que dans l'appendice Les Amours de Milord Édouard Bomston. Seule Julie change radicalement, semble-t-il, et ce à deux reprises : d'abord, dans les premières pages, pour tomber amoureuse de Saint-Preux, puis ensuite, au centre du roman, pour se faire l'épouse fidèle de Wolmar. S'il y a deux grandes parties à la Nouvelle Héloïse, c'est qu'il y a deux grandes transformations dans l'âme de Julie. "Je ne fais point de comparaison entre le commencement et la fin de l'ouvrage. Les détails de la vie domestique effacent les fautes du premier âge : la chaste épouse, la femme sensée, la digne mère de famille font oublier la coupable amante. Mais cela même est un sujet de critique : la fin du recueil rend le commencement d'autant plus repréhensible; on diroit que ce sont deux livres différens que les mêmes personnes ne doivent pas lire ${ }^{62}$. » Plutôt que de la comparer à la Sophie d'Émile, comme on

61. Julie ou la Nouvelle Héloüse, Pléiade II, page 203.

62. Jbid., Seconde Préface, page 17. - Même si ses remarques se trouvent dans la bouche de l'interlocuteur de Rousseau qui joue l'avocat du diable, la critique qu'il fait ici 
le fait d'habitude, on ferait mieux de la rapprocher d'Émile luimême : son existence est l'enjeu du roman et des actions de tous les personnages, c'est sur Julie que l'auteur expérimente et à travers elle surtout qu'il illustre sa pensée sur l'âme humaine.

Cette transformation de Julie permet de comprendre, ce qui est plus important encore, qu'elle est l'équivalent du citoyen dans le Contrat social et d'Émile dans l'Émile. On peut d'ailleurs rapprocher le mode de transformation des individus dans chacun des trois cas. À l'instar du peuple, à l'instar d'Émile, la conversion de Julie est radicale : il s'agit de socialiser un individu naturellement indépendant, voire opposé aux forces sociales et aux valeurs des citoyens ordinaires; semblablement aux deux autres, elle s'opère en bonne partie au moyen d'un revirement religieux. C'est ici le nœud du roman : comment une jeune femme éprise de son amant, rebutée par le mari qu'on lui impose, décidée de tromper ce dernier, comment se transforme-t-elle en honnête épouse? Certes, le processus entier dut être assez long; certes, comme il sera montré plus tard, le mari y fut pour quelque chose. Mais l'essentiel de la transformation, la seule dimension dont Rousseau nous propose le détail se trouve dans la fameuse dix-huitième lettre de la troisième partie. Cette lettre clôt effectivement la première période de la vie de Julie; elle fait le pont avec la seconde et dernière période.

Notons d'abord que la transformation débute dans une église ${ }^{63}$. La décision irrévocable qui fait passer, totalement et définitivement, voudrait-on croire, Julie de l'état d'amante à celui d'épouse se fait par le oui sacramentel. "Quand le Pasteur me demanda si je promettois obéissance et fidélité parfaitte à celui que j'acceptois pour époux, ma bouche et mon coeur le promirent. Je le tiendrai jusqu'à la mort ${ }^{64}$. » A près la cérémonie, c'est vers Dieu que Julie se tourne voyant en Lui, en sa Providence, la cause de son salut moral

souligne une structure dont l'auteur lui-même serait le premier à reconnaître l'importance : l'espace de dix ans qui, dans la logique de l'intrigue, séparent les deux parties n'en serait pas le signe le moins important.

63. "Arrivée à l'Église, je sentis en entrant une sorte d'émotion que je n'avois jamais éprouvée. Je ne sais quelle terreur vint saisir mon ame dans ce lieu simple et auguste, tout rempli de la majesté de celui qu'on y sert. » Ibid., page 353.

64. Ibid., page 354 . 
et, osons le mot, ontologique ${ }^{65}$. Mais quel est le sens de cette transformation? Julie change de vie et d'être parce qu'elle change d'objet d'amour. Plutôt que d'être tournée vers Saint-Preux, plutôt que de vivre d'un amour clandestin et asocial, elle accepte un époux et une famille, elle s'intègre à la société. «Je veux aimer l'époux que tu m'as donné. Je veux être fidèle, parce que c'est le premier devoir qui lie la famille et toute la société ${ }^{66}$. » Sur le socle de cette décision, inspirée en quelque sorte par Dieu, se construira dorénavant la vie légitimée, normalisée et socialisée de Julie ${ }^{67}$, sur le même fondement s'élèvera la deuxième moitié du roman de Rousseau.

Si Julie se rapproche ainsi du citoyen et d'Émile, on trouve sans difficulté dans le roman l'équivalent du législateur du Contrat social et du pédagogue de l'Émile : c'est $\mathrm{M}$. de Wolmar. Certes, il semble se distinguer d'eux en ceci qu'il est athée ${ }^{68}$, mais il leur ressemble profondément selon plusieurs autres aspects. Notons d'abord qu'il partage avec le législateur et le pédagogue cette transcendance qui lui donne des airs de raison incarnée. S'il est humain, Wolmar ne ressent pas les passions humaines; il est

65. «À l'instant, pénétrée d'un vif sentiment du danger dont j'étois délivrée et de l'état d'honneur et de sûreté où je me sentois rétablie, je me prosternai contre terre, j'élevai vers le ciel mes mains suppliantes, j'invoquai l'Être dont il est le trône et qui soutient ou détruit quand il lui plait par nos propres forces la liberté qu'il nous donne. " $\mathrm{Ibid}$., page 356.

66. Ibid., page 357.

67. On pourrait objecter que l'amour de Saint-Preux est semblablement socialisé par les efforts conjugués de Claire, de Wolmar et de Julie, pour ne pas parler de Milord Édouard. Cependant, pour ce qui est de la première moitié du roman, qui ne voit pas que la famille et la société n'ont que peu à faire avec l'amour que Saint-Preux voue à Julie? S'il est question d'enfant, cela vient de Julie et l'enfant n'est qu'un stratagème pour faire accepter leur amour clandestin : jamais n'est-il présenté comme un moyen de « récupérer » l'amoureux et d'en faire un digne époux. Ensuite, pour ce qui est de la deuxième moitié du roman, affirmons tout de suite, ce qui sera expliqué plus loin, que la socialisation et la domestication tentées de l'amour de Saint-Preux se soldent par un cuisant échec. Au contraire, il semblerait que c'est Saint-Preux, le sauvage, qui attire à lui Julie, pourtant socialisée, au moment même où elle lui échappe à tout jamais.

68. Le législateur croit-il vraiment aux dieux auxquels il en appelle pour prouver la bonté de sa législation? Notons d'abord que, du point de vue d'un Rousseau publiquement chrétien, le fait que les législateurs anciens aient fait appel au témoignage des faux dieux païens n'enlève rien à leur grandeur de législateurs, ni à la justesse de leur tactique. Ensuite, on remarquera que le contexte de la présentation de la profession de foi du Vicaire Savoyard éloigne celle-ci du pédagogue d'Émile : l'impression très nette est laissée qu'un pédagogue pourrait utiliser la foi religieuse pour faire faire à son élève des sauts intellectuel et moral absolument nécessaires mais autrement impossibles. 
moins humain que les autres. De mille façons, l'auteur souligne cette caractéristique du personnage. Petit exemple : on ne connaîtra jamais le prénom du «grave Wolmar»; il portera encore moins un surnom aussi romantique que «Saint-Preux». D'ailleurs, il fait sa propre description le mieux du monde: «J'ai naturellement l'âme tranquille et le cœur froid. Je suis de ces hommes qu'on croit bien injurier en disant qu'ils ne sentent rien; c'est à dire, qu'ils n'ont point de passion qui les détourne de suivre le vrai guide de l'homme ${ }^{69}$. » Une seule fois, avoue-t-il, il a senti une émotion: quand il rencontra Julie pour la première fois; une seule fois, reconnâ̂t-il, il a pleuré : au pied du lit de mort de Julie ${ }^{70}$.

Cette maîtrise de lui-même n'a d'égale que sa maîtrise des autres. Car il sait manier, voire manipuler, tous ses serviteurs et servantes avec une dextérité déconcertante par son efficacité et son invisibilité ${ }^{71}$. Car à l'exemple de quelque être divin, il est un parfait connaisseur du cour humain ${ }^{72}$. C'est cette omniscience qui fait de lui le pédagogue d'un peu tout le monde. Car il a formé Julie pour son rôle de mère : ainsi, malgré les apparences, ce n'est pas Julie qui s'est faite elle-même l'éducatrice de ses enfants : elle n'est que l'instrument de Wolmar. Sans doute son incomparable doigté de femme et mère est-il pour beaucoup dans la réussite du projet pédagogique original qui est le leur ; il n'en reste pas moins que le concepteur et l'initiateur de l'ouvrage est l'omniprésent Wolmar ${ }^{73}$.

69. Ibid., page 490 . Voir aussi les pages 487 et 704 .

70. Ibid., pages 492 et 721 . Voir l'avis de Clair sur M. de Wolmar, déjà cité, à la page 203 de la Nouvelle Héloïse

71. Voir les lettres IV, 10 et V, 2. - Rousseau s'efforce en ces lettres, et ailleurs, de reprendre en les développant les thèmes du fameux traité économique de Xénophon. L'Ischomachos de l'Économique de Xénophon devient M. de Wolmar dans le roman. Il est à se demander si, en dernière analyse, Rousseau ne traite pas Wolmar avec une ironie assez semblable à celle de Socrate et de Xénophon envers l'honnête homme athénien qu'ils font parler. Voir Leo STRAuss, Xenophon's Socratic Dialogue, Cornell University Press, Ithaca and London, 1971, 210 pages.

72. Wolmar, qui sait si bien lire dans les cơurs, se décrit mieux que quiconque. «J'aime à lire dans les coeurs des hommes ; comme le mien me fait peu d'illusion, que j'observe de sang-froid et sans intérêt, et qu'une longue expérience m'a donné de la sagacité, je ne me trompe guere dans mes jugemens; aussi c'est là toute la récompense de l'amour propre dans mes études continuelles; car je n'aime point à faire un rolle, mais seulement à voir jouer les autres: La société m'est agréable pour la contempler, non pour en faire partie. » Julie ou la Nouvelle Héloïse, Pléiade II, page 491. On dirait, encore une fois, la description psychologique du législateur du Contrat social ou du pédagogue d'Émile. - Voir aussi pages 487, 496, 609.

73. Voir Ibid., pages 561, 563, 568, 578, 584. 
Il veut même être l'éducateur-législateur de Saint-Preux et, du même coup, de Julie : l'invitation faite à l'amant de vivre à Clarens au sein de sa famille et auprès de son épouse est le premier moment d'une épreuve transformatrice qu'il a imaginée et entreprise en parfaite connaissance de cause. Il en décrit avec aplomb une des étapes à Claire avec des mots qui font voir à quel point il s'imagine le maître du coeur des autres: "En attendant, je cherche à le familiariser avec les objets qui l'effarouchent, en les lui présentant de maniere qu'ils ne soient plus dangereux pour lui. Il est ardent, mais foible et facile à subjuguer. Je profite de cet avantage en donnant le change à son imagination. À la place de sa maitresse je le force de voir toujours l'épouse d'un honnête homme et la mere de mes enfans : j'efface un tableau par un autre, et couvre le passé du présent. On méne un Coursier ombrageux à l'objet qui l'effraye, afin qu'il n'en soit plus effrayé. C'est ainsi qu'il en faut user avec ces jeunes gens dont l'imagination brule encore quand leur cœur est déjà refroidi, et leur offre dans l'éloignement des monstres qui disparoissent à leur approche ${ }^{74}$. $\gg$ 'Il faut bien comprendre qu'il s'agit pour M. de Wolmar non seulement de guérir Saint-Preux, mais aussi Julie. Transcendant par rapport aux passions, le mari de Julie est pour ainsi dire parfaitement immanent: il agit sur les protagonistes avec, semble-t-il, une redoutable efficacité.

D'ailleurs, Saint-Preux ne va-t-il pas jusqu'à comparer Wolmar à Dieu : "Seul entre tous les mortels, il est maitre de sa propre félicité, parce qu'il est heureux comme Dieu même, sans rien désirer de plus que ce dont il jouit : comme cet Être immense il ne songe pas à amplifier ses possessions mais à les rendre véritablement siennes par les relations les plus parfaites et la direction la mieux entendue... ${ }^{75}$ " Terminons en disant tout simplement que c'est toujours avec un brin d'ironie que les amis surnomment SaintPreux «le philosophe»; mais Wolmar reçoit l'épithète «sage» avec tout le sérieux qu'une telle appellation mérite. Il est temps de passer du mari de Julie à son amant.

Lorsqu'on examine les titres des lettres de la Nouvelle Héloïse, on se rend compte que Saint-Preux est le centre occulté de toute la correspondance: lorsqu'il écrit ou reçoit une lettre son nom

74. Ibid., pages 510-511. - Que de morgue dans cette page!

75. Ibid., page 467 . 
n'apparaît jamais contrairement à tous les autres personnages, on sous-entend constamment le nom de Saint-Preux; à l'opposé, les lettres de Julie ou pour Julie, par exemple, indiquent toujours clairement son nom, elle partage en cela le sort de tous les autres personnages ordinaires du roman. C'est là tenter de montrer de façon parfaitement objective, quoique peut-être compliquée, qu'il y a finalement deux centres au roman: Julie et Saint-Preux, le second centre étant moins visible que le premier. Preuve plus simple, Rousseau reconnaît lui-même s'être identifié à SaintPreux lorsqu'il imaginait et écrivait la Nouvelle Héloüse : le point de vue de l'auteur est celui de l'amant, l'origine de son œuvre est sa passion pour les femmes-phantasmes qu'il créait et qui devenaient l'amante et l'amie de Saint-Preux. "Épris de mes deux charmans modéles, je m'identifiois avec l'amant et l'ami le plus qu'il m'étoit possible; mais je le fis aimable et jeune, lui donnant au surplus les vertus et les défauts que je me sentois ${ }^{76}$. " Or qu'en est-il de SaintPreux? Est-il raisonnable comme l'est Wolmar? Est-il réconcilié aux exigences de la vie en société et de la vie de famille comme l'est Julie?

Rien ne permet de l'assurer; au contraire, il faut croire qu'il est un sauvage parmi les civilisés, un passionné parmi les gens raisonnables. Sauvage, il l'est à plus d'un titre. Le signe le plus visible en est le long voyage autour du monde qu'il accomplit pour échapper à la triste Europe, mais sans échapper à l'image de Julie qui le hante. D'ailleurs, sa passion amoureuse est depuis le début une sourde menace pour la civilisation, symbolisée par le père de Julie: ce dernier ne se réconcilie avec lui que lorsqu'il le croit soumis à l'autorité de M. de Wolmar ${ }^{77}$. Mais justement, l' «ancien » amant n'est-il pas toujours épris de Julie? Son périple loin de la civilisation a-t-il éteint sa passion ou n'est-il revenu que plus fidèle? Revenu dans la société européenne, saura-t-il dominer adéquatement son sentiment? Voilà en un sens toute la question du roman; c'est le pari pratique de $\mathrm{M}$. de Wolmar de pouvoir sur ce point précis contrôler l'âme de Saint-Preux. Mais la contrôler n'est pas en anéantir le ressort; et le contrôle qu'impose l'un suppose de l'autre la résistance et la possibilité d'une esquive ou

76. Les Confessions, Pléiade I, page 430

77. Pour le voyage, voir Nouvelle Héloïse, Pléiade II, pages 396;412-415. Pour le sens du personnage de M. d'Étange, voir les pages 349-350 et 605 . 
d'une révolte : pour éloigner tout danger, le mari tout raisonnable devra s'efforcer d'extirper la passion originelle de l'amant ou du moins de la rendre inopérante. Or Rousseau multiplie les indications les plus claires que le feu de l'amour couve toujours dans le cœur de Saint-Preux: chaque fois qu'il se croit purifié, chaque fois qu'il s'affirme guéri pour de bon, un geste, un mot montre au lecteur, et au mari, qu'il n'en est rien ${ }^{78}$. Le dernier signe en est sa résistance au projet de mariage avec Claire que tous : Wolmar, Julie, Claire et Milord Édouard entretiennent pour lui et tentent de lui imposer. Sa réaction est d'une fermeté de roc. Il écrit à Julie dans sa dernière lettre: "Je ne connois rien après vous de si parfait qu'elle; mais fut-elle plus parfaite que vous encore, je sens qu'il faudroit n'avoir jamais été vôtre amant pour pouvoir devenir le sien ${ }^{79}$." Si le mariage est la condition sine qua non de son retour auprès de Julie, il est même prêt à ne plus jamais la revoir. Saint-Preux refuse le mariage de la façon la plus catégorique : ou bien la solitude dans le groupe ou bien la solitude hors du groupe, voilà les seules options qu'il accepte; plus important, il le fait au nom d'un amour qui est la négation de la petite société de Clarens, qu'invente et soutient quotidiennement $M$. de Wolmar.

Cette présentation du personnage de Saint-Preux donne un sens particulièrement prégnant à la dernière lettre de Julie, adressée à son ancien et éternel amant. Le tout dernier paragraphe où Julie tutoie Saint-Preux, ce qu'elle s'empêche bien de faire depuis son mariage, ce qu'elle ne fit jamais avec son mari, montre sans l'ombre d'un doute que l'amour n'a jamais été vaincu non seulement chez l'amant mais aussi chez l'amante: "Adieu, adieu, mon doux ami... Hélas ! j'achève de vivre comme j'ai commencé. J'en dis trop peut-être, en ce moment où le cœur ne déguise plus rien... Eh pourquoi craindrois-je d'exprimer tout ce que je sens ${ }^{80}$ ? " Y avaitil dans ce cœur de femme depuis sa conversion à la vie familiale

78. Voir, par exemple, les pathétiques remarques de Saint-Preux aux pages $421,426,486$, $514-522,527,699,611,764$.

79. Ibid., pages 681-682.

80. Ibid., page 743. - Que cette lettre soit placée après la profession de foi de Julie alors que sa «conversion» avait tant dépendu de la foi et de la religion paraît fort significatif. Voir aussi la page 509 : toute la stratégie de Wolmar, qui visait à s'assurer du cour des deux amants, était vouée depuis le début sinon à l'échec, du moins à des résultats extrêmement incertains. Ce que confirme plusieurs signes avant-coureurs de la reconversion finale. 
une contradiction latente raccordée au prix des plus grands efforts? Sans doute. Mais l'aveu sur le lit de mort, mais l'abandon ultime, mais la reconversion partielle à son premier amour montrent que Rousseau n'oublia jamais une troisième option humaine faite non pas de soumission aux règles du bon sens et encore moins de domination quasi divine de la condition humaine normale, mais de séparation rêveuse et émue. Car à la toute fin du roman, Wolmar se trouve devant non pas un, mais deux cœurs sauvages qui refusent la domestication jusque dans la mort : le dernier mot de Rousseau est à l'effet que la victoire de Saint-Preux le «philosophe » sur le «sage» Wolmar est à peu près totale : malgré les indications les plus sûres, malgré les cautions sociales, Julie ne fut jamais vraiment Mme de Wolmar. Cette chute vers un état présocial ou asocial nourri par un amour clandestin mais irréductible allait connaitre la plus grande fortune dans les cuvres des romantiques. Chez Rousseau, le penseur, elle a l'avantage de soulever l'interrogation première de la philosophie, celle qui porte sur l'homme et son destin.

\section{Conclusion}

L'analyse de ces trois grands textes de Rousseau amène à conclure qu'on trouve chez cet auteur une structure prise et reprise, un triptyque constant. Le citoyen, ou l'homme commun, est débordé dans deux directions : par le sage au-delà de son temps et de sa société, l'homme plus qu'humain, d'une part, et par l'homme avant le temps et la société, l'homme moins qu'humain, d'autre part. Ces trois types humains sont des incarnations des trois conceptions de la vertu qu'on a souvent reconnues coexister chez Rousseau : la vertu-renoncement du citoyen, la vertu-sagesse du philosophe et la vertu-innocence du sauvage. Un seul mot couvrant, trois concepts très différents : voilà l'analogie de base que détaille l'œuvre de Rousseau. En somme, Rousseau - citoyen de Genève, philosophe politique, promeneur solitaire, lequel est le «vrai » Rousseau? - propose des possibilités qui s'entr'appellent en tant que conditions de viabilité les unes des autres, mais ce sont des options peut-être irréconciliables de l'âme humaine. Comme le dit Tzvetan Todorov: "Pour parler de chacune de ces voies, Rousseau se place dans son optique à elle ; pour écarter l'impression de contradiction, il suffit de penser qu'il pratique une sorte de 
«style indirect libre» : il peut dire «je», mais il parle au nom du citoyen, ou de l'homme solitaire, ou de l'homme moral - ce qui n'est pas du tout la même chose. Loin de lui reprocher une contradiction illusoire, nous devons donc lui être reconnaissants d'avoir prêté son génie à ces différentes figures, en nous permettant ainsi de comprendre la logique de chaque position ${ }^{81}$. " Par ailleurs, contre l'analyse de Todorov qui privilégie finalement l'homme moral comme option fondamentale de Rousseau, il nous semble qu'en fin de compte Rousseau opte pour l'homme pré-social ou asocial. Ce n'est pas un hasard, croyons-nous, si on a surtout retenu de la pensée de Rousseau la figure de l'homme dans l'état de nature : ce modèle mi-scientifique mi-imaginatif demeure, malgré les exagérations et les mésinterprétations auxquelles il a prêté flanc, l'approximation la plus juste de la solution finale que nous offre le promeneur solitaire.

Quoi qu'il en soit de cette suggestion finale, et de crainte de paraître durcir une pensée qui est tout sauf rigide, on avouera encore une fois qu'aucun des trois membres de la triade ne peut se concevoir sans les deux autres, mieux : qu'aucun homme, même Rousseau, ne peut se dire hermétiquement l'un ou hermétiquement l'autre. L'important est finalement ailleurs, la question est de dépasser un problème plus ou moins intéressant d'histoire de la philosophie. La condition de ce « dépassement » est d'entreprendre un véritable dialogue avec Rousseau, de le lire avec l'idée qu'il peut nous apprendre quelque chose sur nous, et ce indépendamment de la solution finale qu'il préconise. Lire Rousseau dans cette optique, dialoguer avec lui, c'est reprendre le "Connais-toi toi-même » de Socrate, commandement divin qui, selon ce que raconte le vieux satyre d'Athènes au beau et jeune Alcbiade, résume l'activité philosophique ${ }^{82}$.

Département de philosophie,

Cégep de Ste-Foy

81. Tzvetan Todorov, op. cit., pages 28-29. Voir aussi Leo Strauss, Natural Right and History, University of Chicago Press, 1974, pages 292-294.

82. Platon, Alcibiade premier, 129a, 132c-133c; Xénophon, Les Mémorables, IV. 2,22-40. - Voir aussi, par un recoupement fort intéressant, NIETZSCHE, «La course aux enfers », 408, Opinions et sentences mélées, Denoël/Gonthier, 1902. 